\title{
Geometry of complex instability and escape in four-dimensional symplectic maps
}

\author{
Jonas Stöber ${ }^{1,2}$ and Arnd Bäcker ${ }^{1,2}$ \\ ${ }^{1}$ Technische Universität Dresden, Institut für Theoretische Physik and Center for Dynamics, 01062 Dresden, Germany \\ ${ }^{2}$ Max-Planck-Institut für Physik komplexer Systeme, Nöthnitzer Straße 38, 01187 Dresden, Germany
}

(Dated: September 3, 2020)

\begin{abstract}
In four-dimensional symplectic maps complex instability of periodic orbits is possible, which cannot occur in the two-dimensional case. We investigate the transition from stable to complex unstable dynamics of a fixed point under parameter variation. The change in the geometry of regular structures is visualized using 3D phase-space slices and in frequency space using the example of two coupled standard maps. The chaotic dynamics is studied using escape time plots and by computations of the 2D invariant manifolds associated with the complex unstable fixed point. Based on a normal-form description, we investigate the underlying transport mechanism by visualizing the escape paths and the long-time confinement in the surrounding of the complex unstable fixed point. We find that the escape is governed by the transport along the unstable manifold across invariant planes of the normal-form.
\end{abstract}

\section{INTRODUCTION}

There are different ways in which orbits of a dynamical system may become unstable under variation of some parameter. One famous example is the Hamiltonian-Hopf bifurcation as has first been studied for the triangular equilibrium points of the planar circular restricted threebody problem [1, 2, for which instability occurs beyond a critical mass ratio [3. This is also found for many other examples in celestial and galactic dynamics 4 - 12 for the hydrogen atom 13 15, in the context of molecular dynamics [16, 17, and is also of relevance to particle accelerators [18. The impact of the Hamiltonian-Hopf bifurcation on the phase space geometry has been studied in much detail in Refs. [19]21. Additional insight is provided by computations of invariant manifolds and normal-form descriptions 22 25]. For further results see e.g. Refs. 26 29]. The impact in quantum mechanical systems has been investigated in Ref. [30].

Often its is helpful to reduce the time-continuous dynamics to a discrete-time mapping by means of a Poincaré section. For conservative Hamiltonian systems with three degrees of freedom this leads to the study of four-dimensional (4D) symplectic maps, which are therefore of importance of many areas of physics. Similar to the Hamiltonian case, a transition from stable to complex unstable dynamics is possible for 4D (and higherdimensional) symplectic maps [31, 32]. This has been investigated in detail in the pioneering work 33, 34 for a variant of the 4D coupled standard map [35]. In such a transition to complex unstable dynamics two eigenvalue pairs of the linearized dynamics collide on the unit circle and afterwards form a so-called Krein quartet. This may only happen, if the Krein signature is mixed [31]. A distinctive feature is the spiraling motion in the surrounding of a complex unstable periodic point [6, 30. Moreover, it was found that commonly an extended region around a complex unstable fixed point emerges to which the dynamics is confined for rather long times [11, 3638. Important approaches to understand the complex unstable dynamics are based on computations of the invariant manifolds [36, 38, 39] and normal form descriptions [15, 40, 41. Hamiltonian-Hopf bifurcations have also been studied in much detail for reversible maps, see e.g. Refs. [42, 43].

In this paper, we investigate how the transition from stability to complex instability of a fixed point affects the geometry of invariant objects in its surrounding in the phase space of a 4D symplectic map. This transition is accompanied by the possibility that orbits can escape from the vicinity of the fixed point which is quantified by the average escape times of an ensemble of orbits. The underlying escape mechanism is investigated in terms of the geometry of the stable and unstable manifolds. We provide evidence that the escape occurs across the invariant planes of the normal-form description showing that it is a genuinely higher-dimensional mechanism.

The text is organized as follows. In Sec. II we recall some fundamental properties of linear stability of fixed points and the requirements for complex instability in 4D symplectic maps. Section IIC/summarizes a normal-form description for the transition to complex instability as introduced in Ref. [40]. In Sec. III we introduce a variant of the four-dimensional coupled standard map and define a set of parameters for investigating the transition from elliptic-elliptic stability to complex instability. We visualize the dynamics in the 4D phase space using 3D phasespace slices [44] which is complemented by a frequency space representation [45 47. The escape dynamics is investigated in Sec. IV for an ensemble of initial conditions close to the complex unstable fixed point. To explain the underlying mechanism we compute the stable and unstable manifolds associated with the complex unstable fixed point by utilizing the parametrization method [48 50]. The dynamics of the ensemble suggests that the escape occurs across invariant planes of the corresponding normal-form description. Section $\square$ gives a summary and outlook. 


\section{COMPLEX UNSTABLE DYNAMICS}

\section{A. Linear stability in $4 \mathrm{D}$ maps}

In this section we collect some important results on the stability of fixed points in 4D symplectic maps 31, the Krein collision [31, 51,53 and its normal-form description [40, 54, 55]. A map $\mathcal{M}: \mathbb{R}^{4} \rightarrow \mathbb{R}^{4}$ is called symplectic if its Jacobian matrix $\mathrm{D} \mathcal{M}$ fulfills $\mathrm{D} \mathcal{M}^{T} J \mathrm{D} \mathcal{M}=J$, where $J=\left(\begin{array}{cc}0 & -I \\ I & 0\end{array}\right)$ is the $4 \times 4$ Poisson matrix with $I$ being the $2 \times 2$ identity matrix. An immediate consequence is that a symplectic map is volume preserving as $\operatorname{det}(\mathrm{D} \mathcal{M})=1$. The dynamics in the vicinity of a fixed point, i.e., a point $\boldsymbol{z}^{*}$ that satisfies $\mathcal{M} \boldsymbol{z}^{*}=\boldsymbol{z}^{*}$, is given by the linearized map D $\mathcal{M}$. The symplecticity of $\mathcal{M}$ implies that the characteristic polynomial $P(\lambda)$ of $\mathrm{D} \mathcal{M}$ is reflexive so that coefficients of $P$ come in palindromic form. For a 4D symplectic map this can be written as

$$
P(\lambda)=\lambda^{4}-A \lambda^{3}+B \lambda^{2}-A \lambda+1,
$$

where $A=\operatorname{tr}(\mathrm{D} \mathcal{M})$ and $2 B=A^{2}-\operatorname{tr}\left(\mathrm{D} \mathcal{M}^{2}\right)$. As consequence, the eigenvalues $\lambda_{j}$ with $j \in\{1,2\}$ are restricted to either hyperbolic pairs $\lambda_{j}, \lambda_{j}^{-1} \in \mathbb{R}$, elliptic pairs of $\lambda_{j}, \bar{\lambda}_{j} \in \mathbb{C}$ with $\left|\lambda_{j}\right|=1$ or a Krein quadruplet of complex eigenvalues $\lambda, \lambda^{-1}, \bar{\lambda}, \bar{\lambda}^{-1} \in \mathbb{C}$ with $|\lambda| \neq 1$.

This gives a total of four possible stability types, namely elliptic-elliptic (EE), elliptic-hyperbolic (EH), hyperbolic-hyperbolic (HH) and complex instability (CU). These stability types can be distinguished by introducing the stability index of an eigenvalue pair $\rho=$ $\lambda_{j}+\lambda_{j}^{-1}$ and reducing the characteristic polynomial in Eq. (1) to

$$
R(\rho)=P(\lambda) \lambda^{-2}=\rho^{2}-A \rho+B-2 .
$$

As shown in Ref. [31, different regimes of stability follow from Eq. (2) in dependence on $A$ and $B$. The linearized map D $\mathcal{M}$ is spectrally stable if and only if all roots of $R(\rho)$ are real and within the interval $[-2,2]$. Therefore $R( \pm 2)=0$ yields two stability boundaries, namely

$$
B= \pm 2 A-2 \text {. }
$$

Crossing either of these boundaries corresponds to a saddle-center (SC) or a period-doubling (PD) bifurcation, respectively. Another boundary corresponds to the roots of $R(\rho)$ becoming complex, which occurs when the discriminant of the reduced characteristic polynomial $\Delta R(\rho)=\left(\rho_{1}-\rho_{2}\right)^{2}=0$. This gives the so-called Krein parabola $(\mathrm{KP})$

$$
B=A^{2} / 4+2 .
$$

The possible stability types for an arbitrary fixed point of a 4D map in dependence of $A$ and $B$ can be displayed in the so-called Broucke diagram [1, 31, see Fig. 1. The three stability boundaries SC, PD, and KP lead to

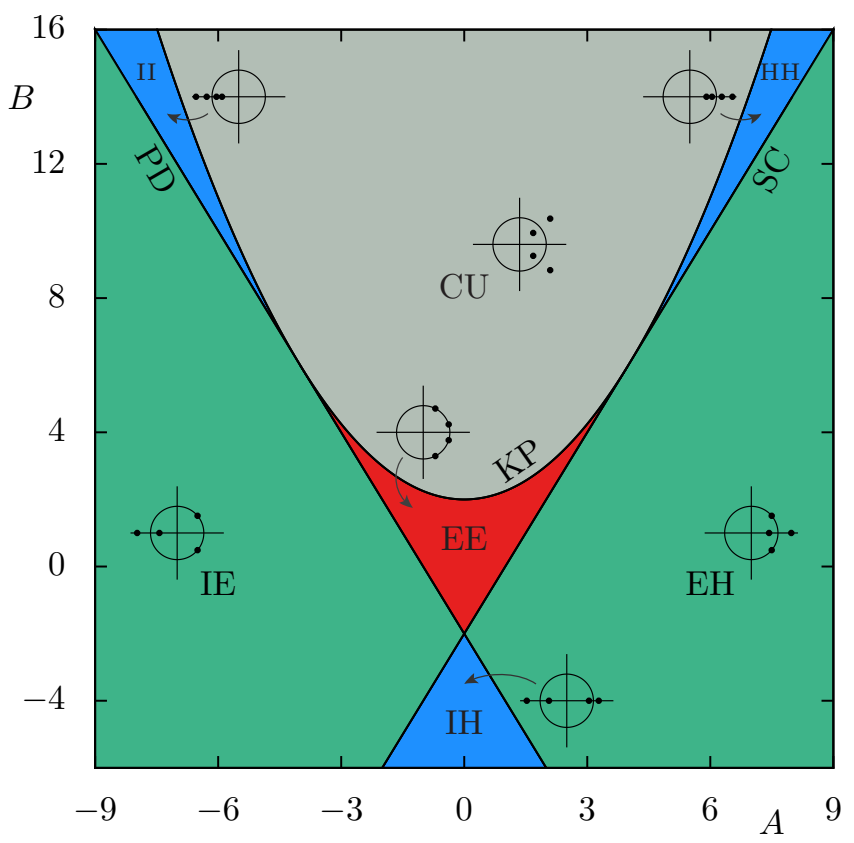

FIG. 1. Stability of a fixed point in dependence on the coefficients $A$ and $B$ of the characteristic polynomial (1) of the linearized map D $\mathcal{M}$. The regions correspond to combinations of elliptic (E), hyperbolic $(\mathrm{H})$, and inverse hyperbolic (I), or complex unstable (CU). The regions are seperated by the period-doubling line (PD), saddle-center line (SC), and the Krein parabola (KP).

seven stability regions corresponding to complex instability (CU) and the different combinations of the elliptic (E), the hyperbolic $(\mathrm{H})$ case, and the inverse hyperbolic (I) case, for which the eigenvalue pair lies on the negative real axis. The corresponding arrangement of the eigenvalues of the linearized map are shown as small insets.

For an EE fixed point the surrounding consists of a two-parameter (Cantor) family of 2D tori as expected from Kolmogorov-Arnold-Moser (KAM) theory. The 2D tori are organized around one-parameter (Cantor) families of elliptic 1D tori. These families are commonly referred to as Lyapunov families, based on the analogy to the Lyapunov center theorem for Hamiltonian flows [56]. Such families of 1D tori have been studied in detail, see e.g. Refs. [36, 57 62. As the families of elliptic 1D tori form the 'skeleton' of the surrounding regular dynamics, they allow for a convenient way to understand the change in geometry occurring when an EE fixed point becomes $\mathrm{CU}$, as will be illustrated below in Sec. IIIB.

\section{B. Krein collision}

As seen from Broucke's diagram in Fig. 1, there are only three possible ways to enter the $\mathrm{CU}$ regime, namely the transition from a) the elliptic-elliptic (EE), or b) the hyperbolic-hyperbolic (HH or II) stability regions through the Krein parabola, or c) through the intersec- 
tion points of the Krein parabola with either the saddlecenter or the period-doubling boundary at $(A, B)=$ $( \pm 4,6)$. The most interesting case is the transition of an elliptic-elliptic fixed point, i.e. case a), as illustrated in Fig. 2 in dependence on some parameter $\alpha$, which controls the transition. For $\alpha>0$, two elliptic eigenvalue pairs approach each other on the complex unit circle until they coalesce at $\alpha=0$. For $\alpha<0$, the eigenvalues split off the unit circle and form a Krein quadruplet.

Whether the eigenvalue pairs of an EE fixed point for a given map can leave the unit circle or pass through each other while staying on the unit circle depends on the socalled Krein signature. This is given by the signature $\left(m_{+}, m_{-}\right)$of the quadratic form

$$
q(x)=x^{T} J \operatorname{D} \mathcal{M} x
$$

which can for example be computed numerically from the eigenvalues of the symmetric matrix $\frac{1}{2}\left(J \mathrm{D} \mathcal{M}+(J \mathrm{D} \mathcal{M})^{T}\right)$, where $m_{+}$is the number of positive and $m_{-}$is the number of negative eigenvalues. If $m_{+}=0$ or $m_{-}=0$ then the fixed point cannot loose stability and stays elliptic-elliptic. Conversely, the fixed point may loose its stability and become complex unstable if the signature is mixed. Note that the quadratic form Eq. (5) allows the construction of an invariant of the linearized dynamics as

$$
q(x)=x^{T} J \mathrm{D} \mathcal{M} x=(\mathrm{D} \mathcal{M} x)^{T} J \operatorname{D} \mathcal{M}(\mathrm{D} \mathcal{M} x)
$$

is preserved under D $\mathcal{M} 33$.

The geometric interpretation of the Krein signature becomes more clear when considering the signature of a multiplier $\lambda$ on the unit circle,

$$
\sigma(\lambda)=\operatorname{sgn} q(u)
$$

where $u$ is any real vector in the eigenspace of $\lambda$. If eigenvalues with the same signature collide on the unit circle, they cannot split off to form a Krein quartet. Explicitly, consider a $4 \mathrm{D}$ symplectic map which is uncoupled, i.e. $\mathcal{M}\left(p_{1}, p_{2}, q_{1}, q_{2}\right)=\left(p_{1}^{\prime}, p_{2}^{\prime}, q_{1}^{\prime}, q_{2}^{\prime}\right)$ with $\left(p_{1}^{\prime}, q_{1}^{\prime}\right)=$ $\mathcal{M}_{1}\left(p_{1}, q_{1}\right)$ and $\left(p_{2}^{\prime}, q_{2}^{\prime}\right)=\mathcal{M}_{2}\left(p_{2}, q_{2}\right)$. Then using the quadratic form (5) and $(1,0,0,0)$ and $(0,1,0,0)$ as vectors of the corresponding eigenspaces the signatures are given by $\sigma\left(\lambda_{i}\right)=\operatorname{sgn}\left(\left(\mathrm{D} \mathcal{M}_{i}\right)_{12}\right)$. Therefore the fixed point can only become complex unstable under some generic coupling if 63, 64]

$$
\operatorname{sgn}\left(\left(\mathrm{D} \mathcal{M}_{1}\right)_{12}\right) \operatorname{sgn}\left(\left(\mathrm{D} \mathcal{M}_{2}\right)_{12}\right)<0 \text {. }
$$

This reflects the counter-rotating nature of the dynamics in the two independent subspaces, similar to the CherryHamiltonian describing two counter-rotating harmonic oscillators 65.

Furthermore, a mixed Krein signature implies that the linearized map of the coalesced eigenvalues has nontrivial Jordan blocks of the shape $m_{+} \times m_{+}$and $m_{-} \times m_{-}$ while the matrix can be diagonalized if the signature

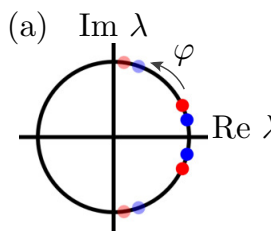

$\alpha>0$

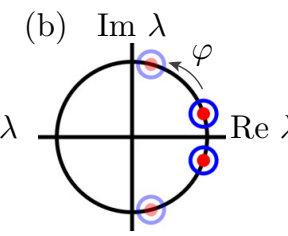

$\alpha=0$

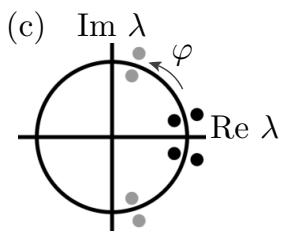

$\alpha<0$
FIG. 2. Krein collision of two elliptic eigenvalue pairs (red and blue circles) in dependence of $\alpha$. The eigenvalues coalesce for $\alpha=0$ and split off the complex unit circle for $\alpha<0$ forming a Krein quadruplet (black circles). For non-zero angle $\varphi$ the location of the Krein collision is move along the unit circle.

is positive or negative definite. Thus, the linearization takes either the form 33

$$
\left(\begin{array}{llll}
\lambda & 1 & 0 & 0 \\
0 & \lambda & 0 & 0 \\
0 & 0 & \bar{\lambda} & 1 \\
0 & 0 & 0 & \bar{\lambda}
\end{array}\right) \quad \text { or } \quad\left(\begin{array}{llll}
\lambda & 0 & 0 & 0 \\
0 & \lambda & 0 & 0 \\
0 & 0 & \bar{\lambda} & 0 \\
0 & 0 & 0 & \bar{\lambda}
\end{array}\right)
$$

where $\lambda=e^{\mathrm{i} \theta}$ and $\left.\theta \in\right] 0, \pi[$. Beside this, in case b) the signature is always mixed. Thus for an II or $\mathrm{HH}$ fixed point there is no constraint to enter the CU region.

\section{Normal form description}

To understand the geometry of regular and invariant structures around a CU fixed point, it is helpful to consider a non-linear normal-form description [40, of which we now summarize the main aspects. Consider a symplectic map $\mathcal{M}$,

$$
\boldsymbol{x}^{\prime}=\mathcal{M}(\boldsymbol{x}, \alpha, \varphi)
$$

with $\boldsymbol{x}, \boldsymbol{x}^{\prime} \in \mathbb{R}^{4}$ and parameters $\alpha, \varphi \in \mathbb{R}$. The fixed point is assumed to be at the origin $\boldsymbol{x}=\mathbf{0}$ such that $\mathcal{M}(\mathbf{0} ; \alpha, \varphi)=0$ for arbitrary $\alpha$ and $\varphi$. Furthermore, the eigenvalues of the linearized map $\mathrm{D} \mathcal{M}(\mathbf{0} ; 0,0)$ are assumed to coalesce ot $\lambda=\exp ( \pm \mathrm{i} \theta)$ with $\theta=2 \pi \nu$ and irrational $\nu \in] 0,1 / 2[$. Note that the case of the rational Krein collision is for example considered in Ref. [55]. The collision is controlled by the parameters $\alpha$ and $\varphi$ as shown in Fig. 2. The parameter $\alpha$ controls the transition from the elliptic-elliptic eigenvalue pair for $\alpha>0$ to the complex unstable quadruplet for $\alpha<0$. The angle $\varphi$ rotates the angle of the Krein collision on the complex unit circle.

In case of the irrational Krein collision with $\alpha=0$ and $\varphi=0$, the linearized map has non-trivial Jordan blocks and can be brought into a Williamson normal-form $L_{0}$ by a symplectic transformation $T$

$$
T^{-1} \mathrm{D} \mathcal{M} T=L_{0}(\mathbf{0} ; 0,0)=\left(\begin{array}{cc}
R_{\theta} & \epsilon R_{\theta} \\
0 & R_{\theta}
\end{array}\right)
$$


where $\epsilon= \pm 1$ and

$$
R_{\theta}=\left(\begin{array}{cc}
\cos \theta & \sin \theta \\
-\sin \theta & \cos \theta
\end{array}\right) .
$$

For $\alpha \neq 0$ and $\varphi \neq 0$, the Williamson normal-form has a transversal two-parameter unfolding, i.e., there is a twoparameter family of matrices that preserve the symplectic form and describes the transition from stability to complex instability via the Krein collision given by [55]

$$
L=L_{0}(\mathbf{0}, \alpha, \varphi)=\left(\begin{array}{cc}
(1-\epsilon \alpha) R_{\theta+\varphi} & \epsilon R_{\theta+\varphi} \\
-\alpha R_{\theta+\varphi} & R_{\theta+\varphi}
\end{array}\right) .
$$

With that, the transformed map $\widetilde{\mathcal{M}}$ in the new coordinates $\boldsymbol{y}$ can be represented as a formal power series

$$
\boldsymbol{y}^{\prime}=\widetilde{\mathcal{M}}(\boldsymbol{y}, \alpha, \varphi) \approx L+\Phi_{2}(\boldsymbol{y}, \alpha, \varphi)+\ldots
$$

where $\Phi_{j}(\boldsymbol{y}, \alpha, \varphi)$ are vector-valued polynomials of degree $j$. In Refs. [40, 54, 55] it is shown that Eq. (14) can be normalized by utilizing a symplectic diffeomorphism $\Psi_{j}(\boldsymbol{y})$ such that $\Psi_{j}^{-1} \circ \widetilde{\mathcal{M}} \circ \Psi_{j}$ is in normal-form with respect to $L$ up to order $j$ for arbitrary $j \in \mathbb{N}$.

As a result, one gets the non-linear normal-form [40]

$$
\left(\begin{array}{l}
x^{\prime} \\
y^{\prime}
\end{array}\right)=\left[\begin{array}{cc}
(1-\epsilon h) R_{\theta+\nu} & \epsilon R_{\theta+\nu} \\
-h R_{\theta+\nu} & R_{\theta+\nu}
\end{array}\right]\left(\begin{array}{l}
x \\
y
\end{array}\right)
$$

with $(x, y)=\left(x_{1}, x_{2}, y_{1}, y_{2}\right) \in \mathbb{R}^{4}$. The parameters

$$
\begin{aligned}
& h=\alpha+b_{1} X+b_{2} I+\ldots \\
& \nu=\varphi+b_{2} X+b_{3} I+\ldots
\end{aligned}
$$

are derivatives of a deduced Hamiltonian generating function with respect to the coordinates $X=x_{1}^{2}+x_{2}^{2}$ and $I=y_{1} x_{2}-x_{1} y_{2}$, respectively. For our purposes we truncate the series of the generating function after quadratic order and obtain $\widetilde{\nu}=\varphi$ and $\widetilde{h} \approx \alpha+b X$ where $\widetilde{h}$ is scaled with respect to $X$ such that $b= \pm 1$.

The normal-form Eq. (15) is guaranteed to be equivariant to a symmetry operation [54, Thm. 2.7], i.e. the normal-form commutes with the action of a symmetry group. A straightforward computation reveals that Eq. 15 is $S^{1}$-equivariant where the symmetry transformation acts as rotation on all coordinates $\left(R_{\gamma} x, R_{\gamma} y\right)$ for $\gamma \in[0,2 \pi[$. The corresponding invariant of Eq. (15) is $I\left(x^{\prime}, y^{\prime}\right)=I(x, y)=y_{1} x_{2}-x_{1} y_{2}$. Consequently, the $4 \mathrm{D}$ nonlinear normal form map can be reduced further by introducing new coordinates.

Hence, we take advantage of the symmetry and visualize the dynamics of Eq. (15) in the hyperplane $x_{2}=0$, see Fig. 3. Note that the full dynamics can be reobtained by applying the symmetry operation, i.e. by simultaneous rotation in the $x$ and $y$ coordinates, see Ref. [40, Eq. (3.1)]. For the sake of clarity, we stick to the half-space with $x_{1} \geq 0$ since the other half can be obtained by the transformation $\left(x_{1}, y_{1}\right) \mapsto\left(-x_{1}, y_{1}\right)$. Furthermore, without loss of generality we fix the parameters $\epsilon=1$ and $b=1$. Firstly, we consider the case
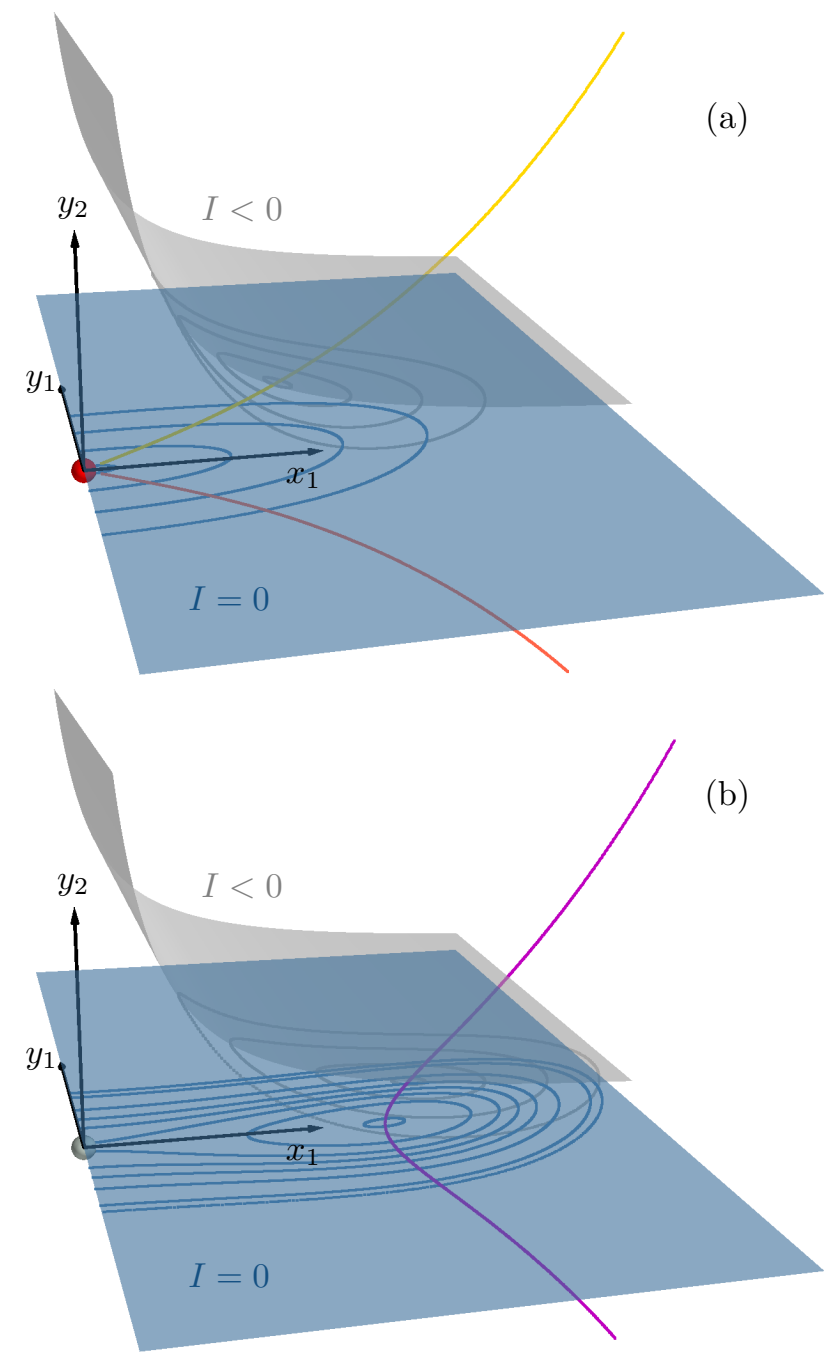

FIG. 3. The reduced Poisson map from Eq. 15 in $\left(x_{1}, y_{1}, y_{2}\right)$ coordinates. The sphere in the origin denotes the trivial fixed point while the gray and the blue planes visualize the $I=$ -0.015 and the $I=0$ plane, respectively. The shown orbits correspond to the same plane as their color indicates. The non-trivial periodic points of the reduced map are depicted as orange and yellow dots for the EE case (a) for $\alpha>0$ and as magenta dots for the CU case (b) for $\alpha<0$.

$I=0$, i.e. $0=I=-x_{1} y_{2}$. Without loss of generality, we choose $y_{2}=0$ and Eq. 15 reduces to a 2D map $f\left(x_{1}, y_{1}\right) \mapsto\left(x_{1}^{\prime}, y_{1}^{\prime}\right)$

$$
\begin{aligned}
x_{1}^{\prime} & =\mid\left(g x_{1}+y_{1} \mid\right. \\
y_{1}^{\prime} & =\left(\widetilde{h} x_{1}-y_{1}\right) \operatorname{sign}\left(g x_{1}+y_{1}\right)
\end{aligned}
$$

with $g=1-\epsilon h$. This map has two periodic points, namely a trivial fixed point at $(0,0)$ which is the original fixed point of $\mathcal{M}$ and for $\alpha<0$ a non-trivial period-two periodic point at $(\sqrt{-\alpha / b}, 0)$. A stability investigation reveals that the trivial fixed point becomes unstable for negative $\alpha$ as expected. In contrast, the non-trivial periodic point only exists when $\alpha \leq 0$ and is always stable. 
This particular situation in the $I=0$ plane corresponds to the typical behavior of a period-doubling bifurcation in a 2D symplectic map, for which a periodic point looses its stability and a stable periodic point of the twice the period is created, see e.g. 66 69].

For the second case $I \neq 0$, the coordinate $y_{2}$ is given by the invariant $I$. Thus, Eq. 15 reduces to a 2D map with all structures living on a hypercolic cylinder $y_{2}=-I / x_{1}$ in the reduced phase space. The map takes the form

$$
\begin{aligned}
x_{1}^{\prime} & =\sqrt{\left(g x_{1}+y_{1}\right)^{2}+I^{2} / x_{1}^{2}} \\
y_{1}^{\prime} & =\frac{\left(g x_{1}+y_{1}\right)\left(y_{1}-\tilde{h} x_{1}\right)+I^{2} / x_{1}^{2}}{x_{1}^{\prime}} .
\end{aligned}
$$

In this case, there is only one non-trivial period-two point, which is given by an implicit equation that we solve numerically.

Figure 3(a) shows the reduced phase space in $\left(x_{1}, y_{1}, y_{2}\right)$ coordinates for $\alpha>0$, i.e., the stable case. The red sphere represents the trivial fixed point which is elliptic-elliptic in this case. The blue and the gray planes as well as the orbits in the same color correspond to $I=0$ and $I=-0.015$, respectively. As long as $\alpha$ is positive, there exits only one fixed point in the $I=0$ plane. For $I>0$ and $I<0$ we get a continuous family of non-trivial periodic points, shown as red and yellow curves, respectively, which are both attached to the trivial fixed point at the origin.

Figure 3(b) shows the reduced phase space for $\alpha<0$. The trivial fixed point (gray sphere) has become unstable and the family of non-trivial periodic points of the $I \neq 0$ plane are detached from the origin similar to a perioddoubling bifurcation in a 2D map. In this way, this family with its surrounding stable 1D tori forms a foliated tubelike object in phase space.

\section{TRANSITION TO COMPLEX INSTABILITY}

\section{A. 4D map with CU fixed point}

The usual 4D standard map 70, 71, which has been investigated in much detail, see e.g. Refs. [37, 44, 7274 , does not allow for CU fixed points. A modified 4D standard map has been introduced in Ref. [33, which is inspired by the Cherry-Hamiltonian describing two counter-rotating harmonic oscillators 65]. As exemplary system to study the transition from EE to CU stability we use a variant of such two coupled counter-rotating 2D standard maps given by the map $\mathcal{M}\left(p_{1}, p_{2}, q_{1}, q_{2}\right) \mapsto$ $\left(p_{1}^{\prime}, p_{2}^{\prime}, q_{1}^{\prime}, q_{2}^{\prime}\right)$ as

$$
\begin{aligned}
p_{1}^{\prime} & =p_{1}+\frac{K_{1}}{2 \pi} \sin 2 \pi\left(q_{1}^{\prime}\right)+\frac{K}{2 \pi} \sin 2 \pi\left(q_{1}^{\prime}+q_{2}^{\prime}\right) \\
p_{2}^{\prime} & =p_{2}+\frac{K_{2}}{2 \pi} \sin 2 \pi\left(q_{2}^{\prime}\right)+\frac{K}{2 \pi} \sin 2 \pi\left(q_{1}^{\prime}+q_{2}^{\prime}\right) \\
q_{1}^{\prime} & =q_{1}+p_{1} \\
q_{2}^{\prime} & =q_{2}-p_{2},
\end{aligned}
$$

where $K_{1}$ and $K_{2}$ are the kicking strengths of the two 2D subsystems and $K$ determines the coupling between them. The phase space is restricted on the torus, i.e. $\left(p_{1}, p_{2}, q_{1}, q_{2}\right) \in\left[-0.5,0.5\left[^{2} \times\left[0,1\left[^{2}\right.\right.\right.\right.$ with periodic boundary conditions. Note that the counter-rotating character of the two uncoupled 2D subsystems in $\left(p_{1}, q_{1}\right)$ and $\left(p_{2}, q_{2}\right)$ is due in the negative sign of the second momentum $p_{2}$ in Eq. (18d) which ensures that condition (8) is fulfilled. This sign is the only difference to the usual 4D standard map, as introduced in Refs. [70, 71]. This map has also been investigated in Ref. [75, though with the negative sign in Eq. 118c instead of Eq. 118d).

We will focus on the central fixed point at $z^{*}=$ $(0,0,1 / 2,1 / 2)$ in the following. Its stability coefficients are

$$
\begin{aligned}
& A=-K_{1}+K_{2}+4, \\
& B=-K_{1} K_{2}+K_{1} K-2 K_{1}+K_{2} K+2 K_{2}+6 .
\end{aligned}
$$

Figure 4 shows the stability diagram for fixed coupling $K=0.1$ in dependence on $K_{1}$ and $K_{2}$. The fixed point is complex unstable in the region between the two straight lines

$$
K_{2}=-K_{1} \quad \text { and } \quad K_{2}=4 K-K_{1} .
$$

The saddle-center and the period-doubling boundaries, Eq. (3), lead to the hyperbolae

$$
-\frac{K K_{1}}{K-K_{1}} \quad \text { and } \quad \frac{-K K_{1}+4 K_{1}-16}{K-K_{1}+4} .
$$

In order to investigate the transition from $\mathrm{EE}$ to $\mathrm{CU}$ stability, we choose the EE region with positive kicking parameters and fix $K_{2}=0.1$ while $K_{1}$ is varied. The six equidistant parameters $K_{1}=0.31,0.305, \ldots, 0.285$, are indicated as black points, labeled by $(\mathrm{A})$ to $(\mathrm{F})$ with (C) lying directly on Krein's boundary, Eq. (4), for $\left(K, K_{1}, K_{2}\right)=(0.1,0.3,0.1)$.

Once the fixed point has become complex unstable, we get a quadruplet of four complex eigenvalues $\left(\lambda, \lambda^{-1}, \bar{\lambda}, \bar{\lambda}^{-1}\right)$ of $\mathrm{D} \mathcal{M}$ where $\lambda=\exp (\beta+\mathrm{i} \theta)$ with $\beta \in \mathbb{R}_{+}$and $\theta \in[0, \pi[$, see Sec. II A. The corresponding eigenvectors $\left(\xi_{1}, \xi_{2}, \bar{\xi}_{1}, \bar{\xi}_{2}\right)$ can be written as $\xi_{j}=u_{j}+\mathrm{i} v_{j}$ with $u_{j}, v_{j} \in \mathbb{R}^{4}$ and $j=1,2$. The stable and unstable invariant subspaces of the linearized map are spanned by $u_{1}, v_{1}$ and $u_{2}, v_{2}$, respectively.

From this one key feature of the dynamics in the surrounding of a $\mathrm{CU}$ fixed point follows: Under the linearized dynamics these eigenvectors evolve as $\xi_{j}^{n}=\lambda_{j}^{n} \xi_{j}$ and consequently provides the evolution in the stable and unstable subspaces by 21 .

$$
\begin{aligned}
& u_{j}^{(n)}=\exp ( \pm \beta n)\left(\cos (n \theta) u_{j}-\sin (n \theta) v_{j}\right) \\
& v_{j}^{(n)}=\exp ( \pm \beta n)\left(\sin (n \theta) u_{j}+\cos (n \theta) v_{j}\right)
\end{aligned}
$$

where the positive sign corresponds to $j=1$ and the negative to $j=2$. Any point $z$ in the $4 \mathrm{D}$ phase space can be expressed in the basis of the eigenvectors, i.e. $z=$ 


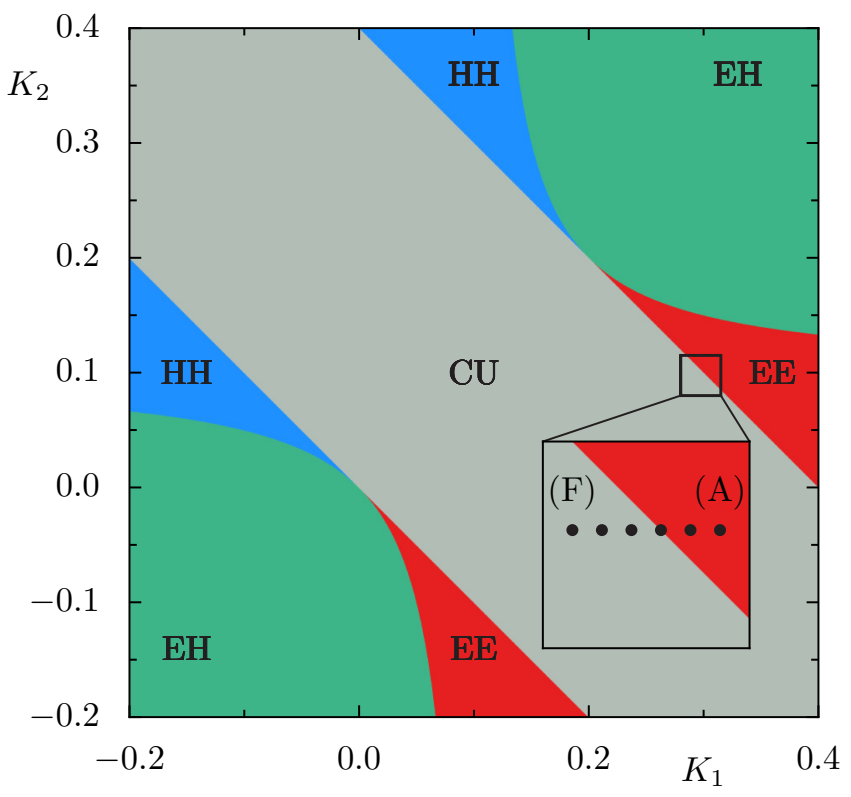

FIG. 4. Stability of the fixed point $(0,0,1 / 2,1 / 2)$ for fixed $K=0.1$ in dependence of $K_{1}$ and $K_{2}$. The magnification shows the selected parameters for the transition from EE to CU, (A) $K_{1}=0.31$, (B) $K_{1}=0.305, \ldots$, and (F) $K_{1}=0.285$.

$c_{1} u_{1}+c_{2} v_{1}+c_{3} u_{2}+c_{4} v_{2}$ with coefficients $c_{1}, c_{2}, c_{3}, c_{4} \in \mathbb{R}$. These coefficients can be determined with the help of the basis of the dual space of the matrix of eigenvectors 44 . Using the time evolution of the eigenvectors Eq. 22 allows for obtaining the linearized dynamics of an orbit for a given initial condition. Apparently, the underlying dynamics is governed by an expanding/contracting part and a rotating part which leads to a spiraling motion as illustrated in Fig. 5. If $c_{1}$ or $c_{2}$ are different from zero, the expanding dynamics will asymptotically dominate. Note that this provides a good description for some limited number of iterations of the map $\mathcal{M}$ only, beyond which the nonlinear dynamics becomes relevant, as can be seen by the deviations between the real orbit depicted as colored spheres and the linerized dynamics shown as black curve in Fig. 5 .

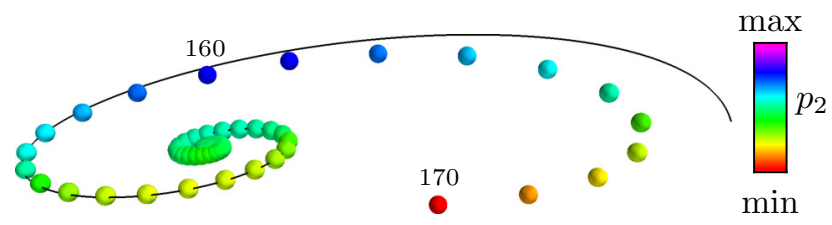

FIG. 5. Spiraling motion of an orbit started close to the CU fixed point. Shown are the $\left(p_{1}, q_{1}, q_{2}\right)$ coordinates with $p_{2}$ encoded in color of the first 170 iterates of the point $(0,0,0.5,0.5)+\mu$ for $\mu=10^{-8}$. The initial spiraling motion is well described using the linearized dynamics, Eq. 22 , as shown by the black curve. From the 160th iterate deviations become visible in the plot.

\section{B. 3D phase-space slice}

To get an intuition for the dynamics of the transition from EE to CU stability of the fixed point in phase space, we use a 3D phase-space slice [4]. The idea is to reduce the $4 \mathrm{D}$ phase space by one dimension by considering a 3D hyperplane $\Gamma$ and determining those points of an orbit that fulfill the slice condition

$$
\Gamma_{\varepsilon}=\left\{\left(p_{1}, p_{2}, q_{1}, q_{2}\right)|| p_{2} \mid \leq \varepsilon\right\} .
$$

For the resulting points the coordinates $\left(p_{1}, q_{1}, q_{2}\right)$ are displayed in a 3D plot. The parameter $\varepsilon$, i.e., the thickness of the slice, controls the resolution. Smaller values of $\varepsilon$ require longer orbits to obtain the same number of points in the slice as the slice condition (23) is fulfilled less often. For all 3D phase-space slice plots in this paper we choose $\varepsilon=10^{-6}$. Typically $f$-dimensional objects in the full 4D phase space appear as $(f-1)$-dimensional objects in the 3D phase-space slice. For example 2D tori lead to two (or more) separate (but dynamically connected) rings in the 3D phase-space slice and 1D tori lead to two (or more) points in the slice. For further examples, also including more general slice conditions, and detailed discussions see Refs. 44, 61, 62, 75, 78.

Figure 6 shows a sequence of 3D phase-space slice plots of regular orbits in the vicinity of the central fixed point for the parameter sets $(\mathrm{A}),(\mathrm{C})$, and $(\mathrm{F})$, see Fig. 4 . In Fig. 6(a) for parameter set (A), i.e. $K_{1}=0.31$, one is in the stable regime and quite far away from the Krein collision. The EE fixed point (red sphere) is surrounded by regular 2D tori shown as grey curves, which form pairs closed loops on either side of the fixed point. The general arrangement of the 2D tori is governed by the two (Lyapunov) families of 1D-tori which are attached to the EE fixed point and shown in yellow and orange, respectively. Due to the symmetries of the map, both families lie in the $q_{1}-q_{2}$ plane. Thus they can be displayed in 2D diagrams to clarify the change of the families under parameter variation, see Figs. 6(d)-(f). Note that the small gap in the yellow family in Fig. 6(a) is caused by a resonance, see Sec. IIIC Both families of elliptic 1D-tori are surrounded by regular 2-tori which form pairs of rings in the 3D phase-space representation, depicted in gray color. Interestingly, the regular 2 -tori in the direct vicinity of the fixed point show a strong bending close to the fixed point. This geometry is similar to the phase space of the normal-form for $\alpha>0$ in Fig. 3(a) where the hyperbolic shape of the $I \neq 0$ plane forces the tori to bend away from the $y_{1}-y_{2}$ plane. Furthermore, the families of 1D tori correspond to the family of period-two periodic points in the normal-form.

Figure 6(b) shows the situation for point (C) in parameter space with $K_{1}=0.3$. For this parameter the two eigenvalue pairs of the linearized map at the fixed point coalesce at two places on the complex unit circle, see Fig. 2(b). When approaching the Krein collision parameter, the angle between the eigenvectors of the linearized map decreases until the eigenvectors of the eigenvalue 

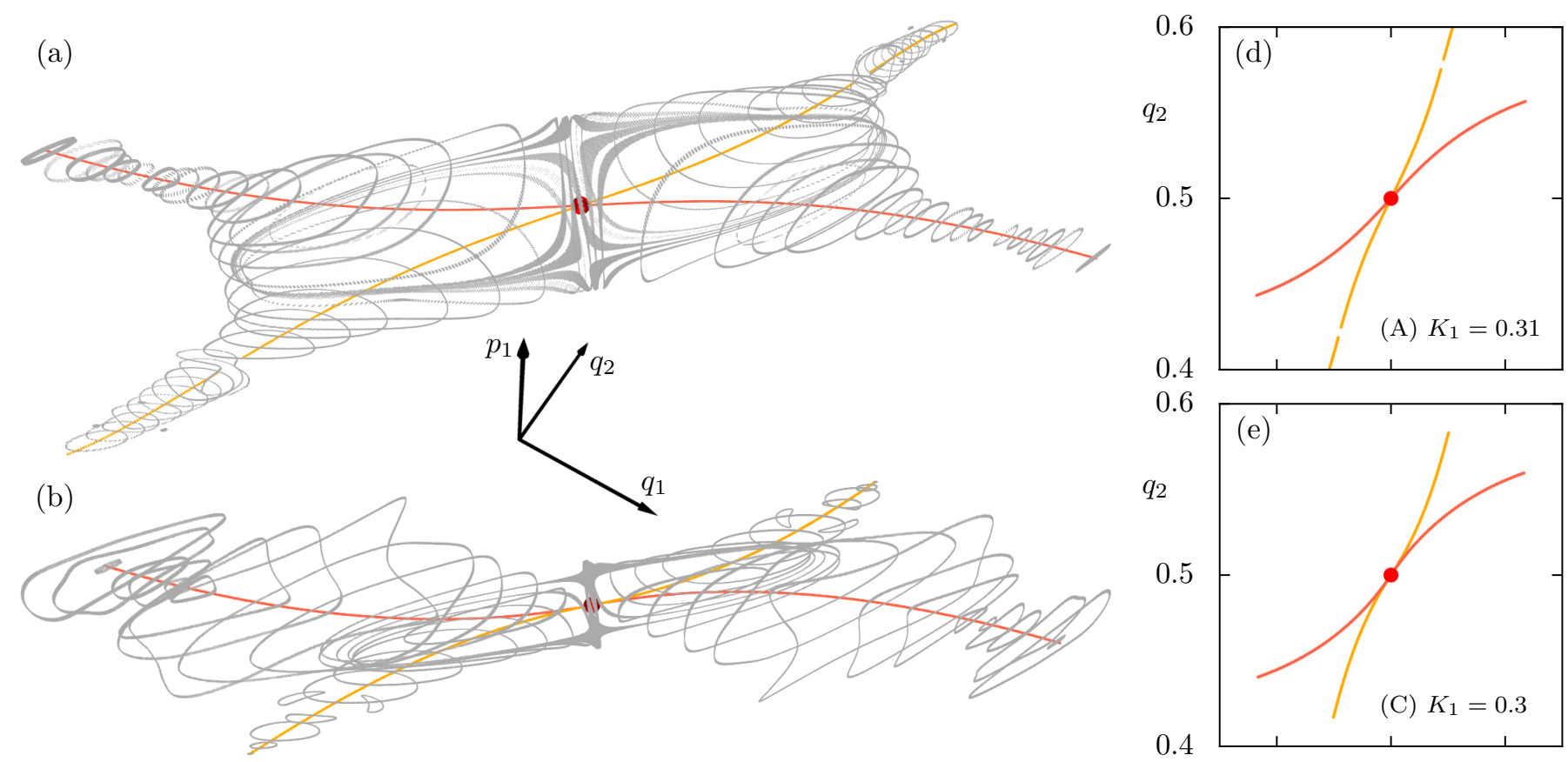

(c)
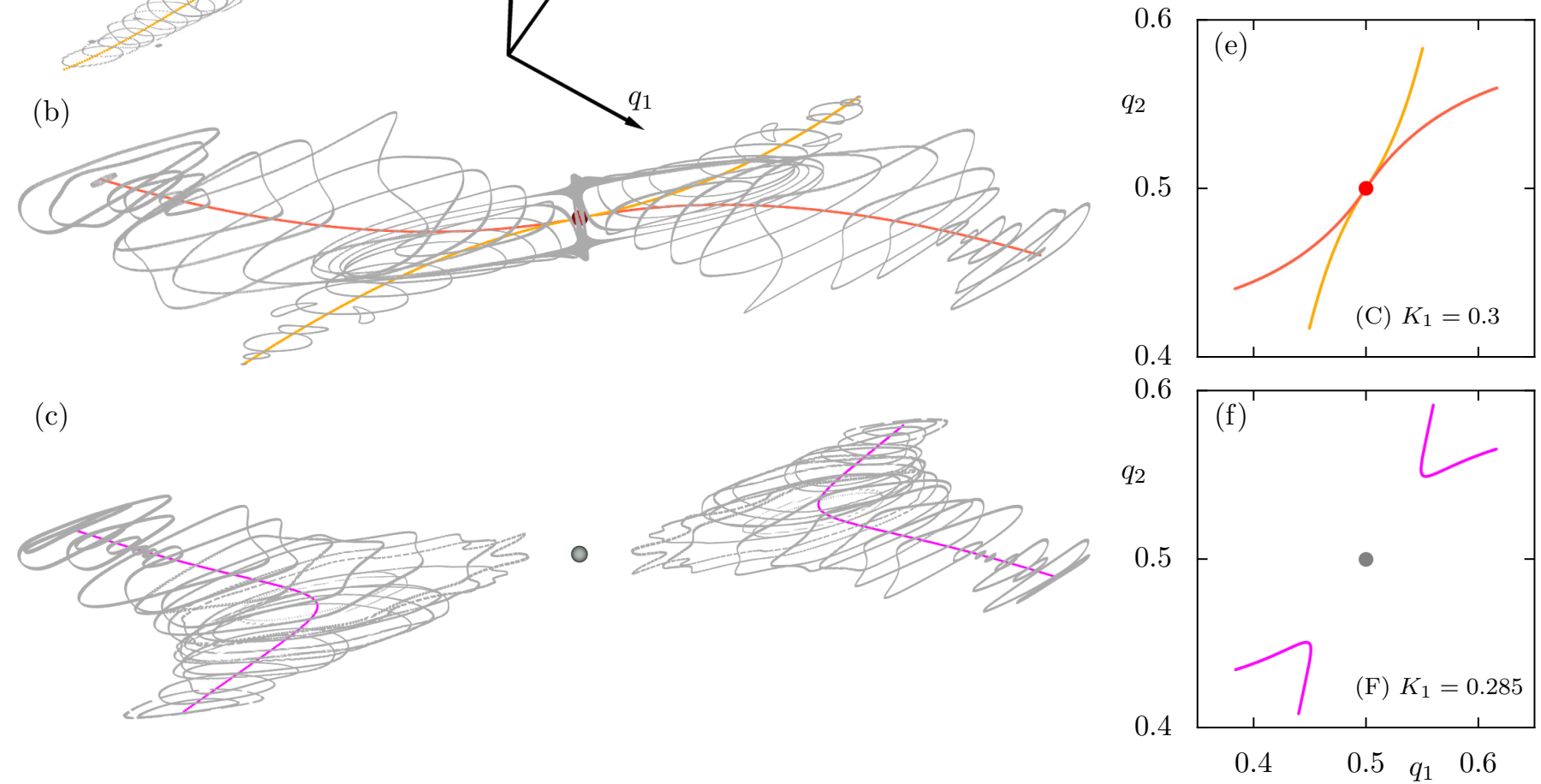

FIG. 6. Sequence of 3D phase-space slice plots of regular tori represented as grey rings in the vicinity of the fixed point shown as red spheres for elliptic-elliptic stability and as a grey sphere for complex instability. The families of 1D tori (red, yellow, magenta) form the skeleton of the surrounding $2 \mathrm{D}$ tori. The chosen parameters are (a) $K_{1}=0.31$, (b) $K_{1}=0.3$, (c) $K_{1}=0.285$ and correspond to points $(\mathrm{A}),(\mathrm{C})$ and $(\mathrm{F})$ in parameter space, see Fig. 4 The right column (d), (e), (f) depicts the families of $1 \mathrm{D}$ tori, which lie in the $q_{1}-q_{2}$ plane due to the symmetry of the map. For a rotating view see http://www.comp-phys.tu-dresden.de/supp/.

pairs become collinear. Accordingly, the families of 1D tori are approximately parallel in the vicinity of the fixed point as can be seen in Fig. 6(b).

Finally, Fig. 6(c) shows the situation after the Krein collision, i.e. for $K_{1}=0.285$, which corresponds to parameter (F) in Fig. 4. Once the fixed point has become complex unstable, the two families of 1D tori detach from the fixed point and merge into one single family. This corresponds to the normal-form behavior for $\alpha<0$, see Fig. 3(b). The regular tori close to the family of 1D tori persist. Interestingly, orbits in the vicinity of the $\mathrm{CU}$ fixed point stay in its surrounding for very long times and only eventually escape. This will be discussed in more detail in Sec. IV.

To quantify the detachment of the regular 2D tori from the $\mathrm{CU}$ fixed point, we compute the minimal distance $d_{\text {tori }}$ between the complex unstable fixed point and the family of 1D tori. In the normal-form description of Sec. IIC the minimal distance is given by the distance between the trivial fixed point at the origin and the periodtwo periodic point, namely by $d_{\text {tori }}=\sqrt{-\alpha} / b$. For the 4D map this translates in first approximation to

$$
d_{\text {tori }} \propto \sqrt{K_{1}^{*}-K_{1}}
$$

with $K_{1} \leq K_{1}^{*}=0.3$. Figure 7 shows the numerically determined minimal distance $d_{\text {tori }}$ in dependence on the kicking strength $K_{1}$ as black dots. Good agreement with the square root behavior (24), shown as a dashed line, is found. Further away from the Krein collision parameter small deviations become visible. 


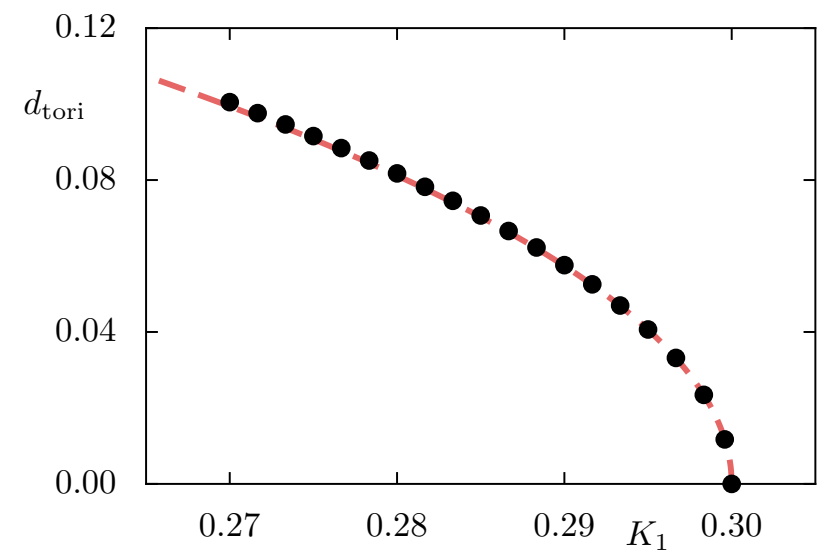

FIG. 7. Minimal distance $d_{\text {tori }}$ between the central fixed point and the family of 1D tori in dependence on $K_{1}$. The distance follows the predicted behavior $\propto \sqrt{K_{1}^{*}-K_{1}}$, shown as red dashed line for $K_{1} \leq K_{1}^{*}=0.3$.

\section{Frequency space}

Complementary to the representation in phase space one can display regular tori in frequency space, which is particularly useful for understanding the influence of resonances. A regular torus is characterized by two frequencies, one describing the motion along the major radius of the 2-torus and one for the motion along the minor radius. Numerically the frequencies $\nu_{1}, \nu_{2} \in[0,1[$ for an orbit started in a phase-space point $\left(p_{1}(0), p_{2}(0), q_{1}(0), q_{2}(0)\right)$ are determined using a Fouriertransform based frequency analysis [45, 46, 79, 80]. As signals $z_{j}(n)=q_{j}(n)-\mathrm{i} p_{j}(n)$ for each degree of freedom $j=1,2$ is used, where $\left(q_{j}(n), p_{j}(n)\right)$ are the coordinates obtained from $N$ successive iterates of the map. In order to distinguish regular and chaotic motion, the frequencies $\nu_{j}$ of the first half of an orbit, i.e., iterates in the interval $n \in[0, N / 2-1]$, are computed and compared to the frequencies $\widetilde{\nu}_{j}$ of the second half, i.e., the iterates in the interval $n \in[N / 2, N]$. For the motion on a regular torus, the difference of these frequency pairs should be rather small. Thus if the maximal difference $\max \left\{\left|\nu_{j}-\widetilde{\nu}_{j}\right|\right\}$ is smaller than some threshold $\delta_{\text {cut }}$, we consider the orbit as regular. In the following $\delta_{\text {cut }}=10^{-8}$ is used. Of course, such a numerical criterion does not guarantee that the orbit eventually could become chaotic at very large times, as is also the case with other chaos indicators, see Ref. 81 for a recent overview. Using an ensemble of $10^{7}$ initial conditions, randomly chosen in the $4 \mathrm{D}$ phase-space volume defined by $p_{1}, p_{2} \in[-0.1,0.1]$ and $q_{1}, q_{2} \in[0.4,0.6]$, and plotting the frequencies $\left(\nu_{1}, \nu_{2}\right)$ of the regular tori provides the two-dimensional frequency space representation.

Figure 8 shows a sequence of such frequency space plots for all six parameter sets specified in Fig. 4. The frequencies of the EE fixed point is indicated by a large red point in Fig. 8(a)-(c). For the complex unstable fixed point there is only one frequency given by the angle of the complex eigenvalues, which is shown on the $-1: 1: 0$ resonance line as large grey point in Fig. 8(d)-(f). Although hardly noticable, the angle gets smaller with decreasing $K_{1}$. As for the 3D phase-space slice shown in Fig. 6, the orange, yellow and magenta points mark the frequencies of the families of 1D tori, which form the edges of the gray regions of regular tori.

Resonances correspond to straight lines in frequency space,

$$
n_{1} \nu_{1}+n_{2} \nu_{2}=m
$$

with $m, n_{1}, n_{2} \in \mathbb{Z}$ and $\operatorname{gcd}\left(m, n_{1}, n_{2}\right)=1$ and either $n_{1} \neq 0$ or $n_{2} \neq 0$. Some relevant resonance lines are shown as blue dashed lines, labeled by $n_{1}: n_{2}: m$. Such resonances lead to resonance channels [46] and gaps in the families of $1 \mathrm{D}$ tori 61 .

The typical frequency space around an EE fixed point is seen in Fig. 8(a)-(b) for $K_{1}=0.31$ and $K_{1}=0.305$ which corresponds to parameters (A) and (B) in Fig. 4. respectively. Both families of 1D tori are attached to the fixed point forming a cusp and the regular tori fill a region in between these families. As the eigenvalues approach the Krein collision parameter in Fig. 8(b), the fixed point has to approach the $-1: 1: 0$ resonance line since the eigenvalues of the linearized map eventually coalesce on the pair $\mathrm{e}^{ \pm \mathrm{i} 2 \pi \nu}$ with $\nu=\nu_{1}=\nu_{2}$. This shift of the frequencies of the fixed point stretches the families of 1D tori and the top of the cusp accordingly. During this process, the density of regular tori close to the $-1: 1: 0$ resonance line decreases. This becomes especially apparent in case of the Krein collision parameter in Fig. 8(c), i.e. for parameter $(\mathrm{C})$ in Fig. 4 for $K_{1}=0.3$. This corresponds to the tangency of the families of $1 \mathrm{D}$ tori so that only few regular tori exist in the surrounding of the fixed point.

Figure 8(d)-(f) show the frequency space plots for the complex unstable case for $K_{1}=0.295, K_{1}=0.29$, and $K_{1}=0.285$, corresponding to the points (D), (E) and (F) in Fig. 4. The two former families of 1D tori merge in the Krein collision parameter and subsequently detach from the $-1: 1: 0$ resonance line once the fixed point looses its stability. We observe two branches of the merged family which bend away from the fixed point and simultaneously from the resonance line. Note, that these branches are actually connected which can be seen by applying the unimodular transformation $\left(\nu_{1}, \nu_{2}\right) \mapsto\left(\nu_{2}, 2 \nu_{2}-\nu_{1}\right)$ to the upper branch resulting in the magenta dotted line. The transformed branch connects seamlessly to the other branch yielding a complete arc beginning and ending at $\nu_{2} \approx 0$. This illustrates that both branches actually belong to just one family of 1D tori after the fixed point has turned CU. In general, such linear transformations with determinant \pm 1 can always be applied for systems of periodic functions [82, Theorem 5 and 6].

Shortly after the transition of the fixed point to complex instability, there are no regular tori in its vicinity or the $-1: 1: 0$ resonance line in frequency space. How- 

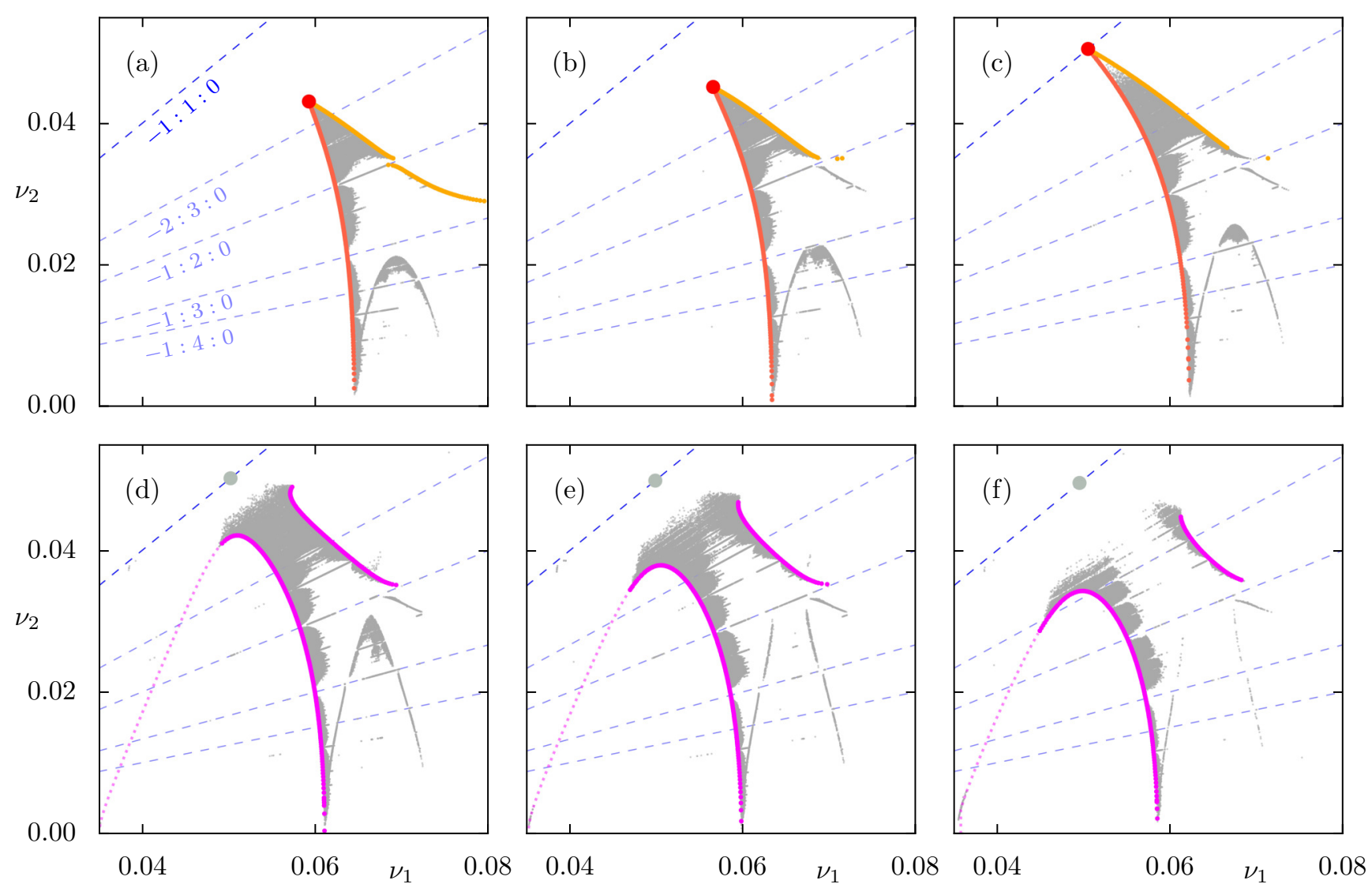

FIG. 8. Frequency space for different parameters $K_{1}$ : (a) $K_{1}=0.31$, (b) $K_{1}=0.305$, .., and (f) $K_{1}=0.285$, corresponding to (A) $-(\mathrm{F})$ in Fig. 4 . Light gray dots correspond to regular orbits while the orange, yellow, and magenta dots correspond to the families of 1D tori. The frequency of the elliptic-elliptic (red) and complex unstable (gray) fixed point are depicted as enlarged dot. The dotted magenta curve in (d)-(f) is the unimodular transformation of the upper branch of 1D tori. Some relevant resonance lines are shown as dashed lines.

ever, the regular tori between the branches of the former cusp still exist directly after the transition as is visible in Fig. 8(d). Only when the fixed point becomes more unstable, the distance of the branches increases and the density of regular tori between them decreases until a gap emerges, see Fig. 8(f). The remaining regular orbits in Fig. 8(f) are close to the family of 1D tori. This confirms the observations in the 3D phase-space slice in Fig. 6(c), where regular tori are only found in the surrounding of the family of $1 \mathrm{D}$ tori and no regular structures are left in the direct vicinity of the fixed point.

Note that the arc like structure in the range of $0.06 \leq$ $\nu_{1} \leq 0.075$ below the discussed region of regular tori, see Fig. 8(a)-(d), belongs to regular orbits in the surrounding of a periodic orbit close to the central fixed point. Although these orbits are not in the focus of this study they illustrate how the complex instability of the fixed point gradually destroys all stable structures in its vicinity.

\section{ESCAPE FROM THE CU REGION}

When the EE fixed point becomes CU, this immediately affects its direct surrounding as the two elliptic families of 1D tori become detached from the fixed point. Thus there are also no regular tori in its direct vicinity. Instead one has a 2D stable and a 2D unstable manifold which lead to chaotic dynamics. However, in practice close to the Krein collision parameter, initial conditions in the vicinity of the fixed point lead to orbits staying for very long times in a confined phase-space volume. In this section, we investigate this behavior and the underlying escape paths in more detail.

\section{A. Escape times}

To study the escape of orbits from the surrounding of the $\mathrm{CU}$ fixed point, we use escape time plots as in Refs. [75, 83, 84, see Fig. 9. Using a grid of initial conditions on a particular plane in the 4D phase space for each initial point the escape time $n_{\text {esc }}$, required to reach some specific exit region, is determined. Since we are in- 

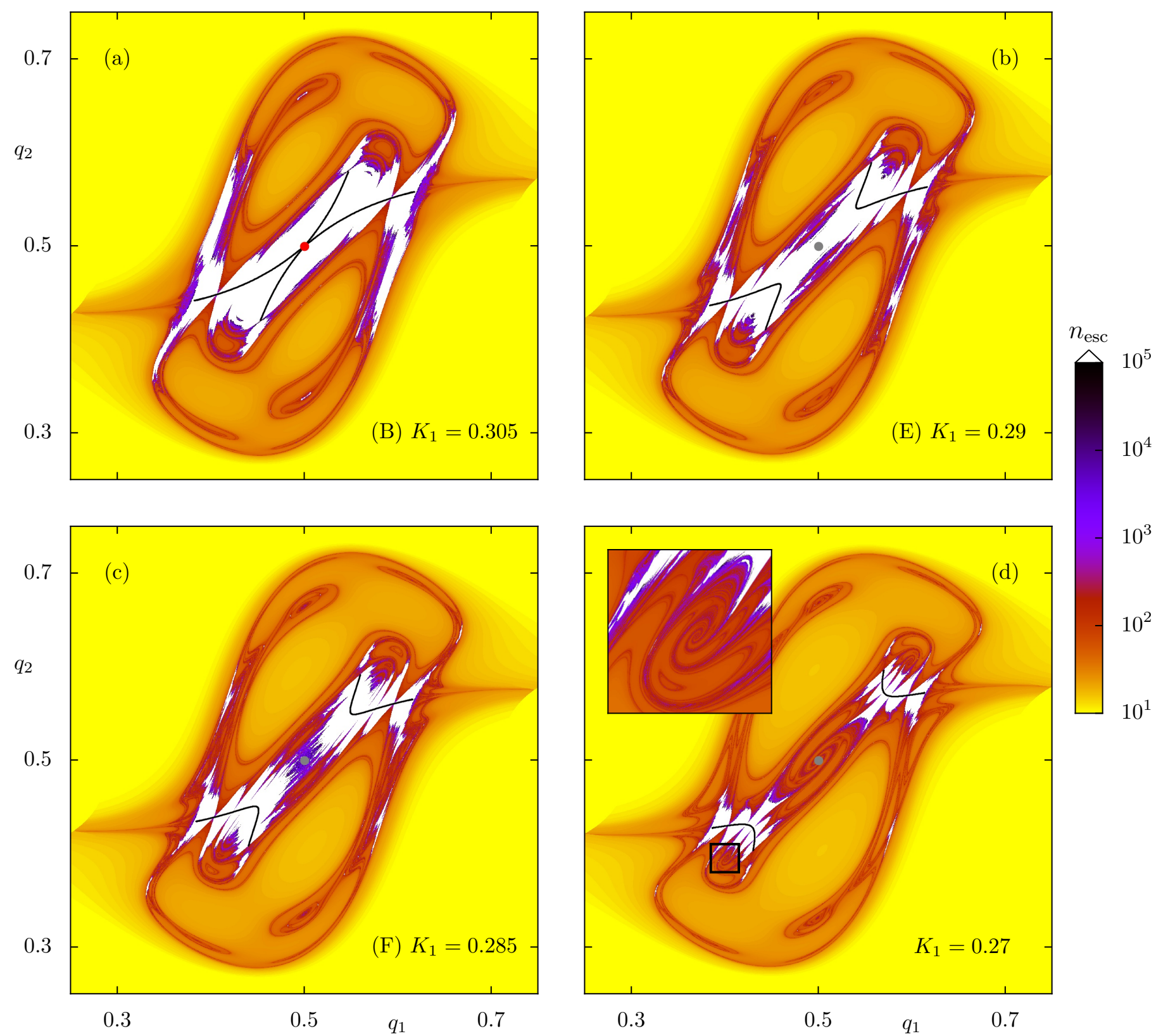

FIG. 9. Escape time plots in the $q_{1}-q_{2}$ plane for $p_{1}=0$ and $p_{2}=0$ for (a) $K_{1}=0.305$, (b) $K_{1}=0.29$, (c) $K_{1}=0.285$, and (d) $K=0.27$. The escape time is encoded in color, where white corresponds to those points which have not escaped within $n_{\max }=10^{5}$ iterations. The fixed point is shown as red (elliptic-elliptic) or gray dot (complex unstable) and the families of $1 \mathrm{D}$ tori are shown as black dots.

terested in the behaviour close to the family of 1D tori, we choose the initial points in the $q_{1}-q_{2}$ plane through the fixed point with $q_{1}, q_{2} \in[0.25,0.75]$ and $p_{1}=0$ and $p_{2}=0$. On this plane, a $2000 \times 2000$ grid of initial points is used. We define the exit region as $q_{1}, q_{2} \notin[0.25,0.75]$ with arbitrary momenta $p_{1}, p_{2} \in[-0.5,0.5]$. Each initial condition is iterated until it enters the exit region or a maximal number $n_{\max }$ of iterations is reached.

Figure 9 shows the escape time $n_{\text {esc }}$ encoded in color ranging from yellow for fast escaping points to black for nearly regular orbits while white points do not escape to the exit region within $n_{\max }=10^{5}$ iterations (though they may escape eventually). In addition, the families of 1D tori are shown in black and the fixed point as red or gray dot for $\mathrm{EE}$ or $\mathrm{CU}$ stability, respectively. The parameters for Fig. 9(a)-(c) correspond to the points (B), $(\mathrm{E})$, and $(\mathrm{F})$ in parameter space specified in Fig. 4. In addition, the families of 1D tori are shown in black and the fixed point as red or gray dot for EE or CU stability, respectively.

As before, we focus on the structures close to the fixed point. For the EE case, the vicinity of the fixed point is 
naturally governed by a white region which corresponds to the regular 2-tori surrounding the families of 1D tori, compare with Fig. 9(a). Thus, even for arbitrarily large times these orbits do not escape. Furthermore, we see the impact of the $-1: 3: 0$ resonance in form of a notch in the white region. This is consistent with the frequency analysis in Fig. 8(a) for $K_{1}=0.31$.

If the eigenvalues of the fixed point approach the Krein collision parameter the fraction of the white points only slightly diminishes and the overall pattern of the escape time plot does not change much (not shown). After the transition to complex instability, see Figs. 9(b)-(d), the white region reduces substantially. Starting with the appearance of two small unstable regions in the white region for $K_{1}=0.29$ in Fig. 9(b) above and below the fixed point. Still, there are orbits in the direct vicinity of the fixed point which stay close to it for more than $n_{\max }$ iterations. Quantitatively, the size of the white region depends on the threshold $n_{\max }$, but a larger value of $n_{\max }$ does not affect the shown escape time plots significantly. The reason for this is that orbits in the vicinity of the CU fixed point are confined for an extremely long time when the parameters of the map are sufficiently close to the EE region in Fig. 4. The more unstable the fixed point becomes, i.e. the smaller $K_{1}$ is, the more the two branches of the family of $1 \mathrm{D}$ tori separate and the white region diminishes because the regions of instability get larger. Finally, for point (F) in Fig. 4 with $K_{1}=0.285$ all orbits in the direct vicinity of the fixed point are able to reach the exit region within $n_{\max }$ iterations, see Fig. 9 (c). For this parameter we observe that the unstable regions in the escape plots reach the fixed point, and consequently the large white region is divided into two smaller ones. These two white regions correspond to the tubes of regular motion in the 3D phase-space slice representation, e.g. see Fig. 6(f), as well as the attached regular tori of the branches of the family of 1D tori in frequency space, see Fig. 8(f).

Figure 9(d) shows the escape time plot for $K_{1}=0.27$, i.e. far in the $\mathrm{CU}$ regime. The branches of the family of 1D tori moved far away from the fixed point and the unstable region in between is large. Interestingly, this unstable region reveals a unique spiral pattern which is attached to the fixed point. Orbits on this spiral need at least one to two orders of magnitude more iterations to escape into the exit region than the neighboring ones. Additionally, there is another spiral structure on a smaller scale as shown in the magnification in the inset.

A closer investigation of orbits started in the darker colored region reveals that the spiral pattern is due to the influence of the $-2: 3: 0$ resonance: In cases where a frequency analysis of these orbits is possible, i.e. the orbit is confined for long times and considered as regular by our algorithm, see section III C, we get frequencies on or close to this resonance line.

The escape time plots raise the following question: Which structures govern the slow transport in the vicinity of a complex unstable fixed point? An important ingredient to answer this question are the invariant manifolds of the fixed point, which are discussed in the next section.

\section{B. Stable and unstable manifolds}

The stable and unstable manifolds associated with an unstable fixed point govern the chaotic dynamics in its surrounding. For a complex unstable fixed point of a 4D map the manifolds are two-dimensional invariant objects in the 4D phase space. Numerically the manifolds are computed using the parameterization method 48, 50, 77, 85, 86, see Appendix A for details. In the 3D phasespace slice representation they lead to one-dimensional curves, see Fig. 10, where the red curve corresponds to the unstable manifold and the blue curve to the stable manifold.

The regular 2-tori (gray loops) as well as the families of 1D tori (black curves) in Fig. 10(a) are the same as in Fig. 6(f). Figure 10(b) shows the geometry for a smaller value of $K_{1}=0.28$. The complex unstable fixed point is indicated by a gray sphere in both plots.

Numerically it is found that the stable and unstable manifolds intersect in one point. This point therefore is a homoclinic point whose forward iterates approach the fixed point on the stable manifold while the backward iterates approach the fixed point on the unstable manifold. The existence of a transverse homoclinic point therefore immediately implies an infinity of such homoclinic points. Note that generically two 2D manifolds in a 4D phase space will not intersect. The fact that this happens for the manifolds of the considered fixed point must be due to the symmetries of the map.

The geometry becomes more clearly visible for smaller $K_{1}=0.28$ as shown in Figure 10 (b). The arrangement of the manifolds in the $3 \mathrm{D}$ phase-space slice reminds of the homoclinic tangle in 2D symplectic maps. In comparison to Fig. 10(a) the excursions of the manifolds are more pronounced which corresponds to a larger chaotic region surrounding the complex unstable fixed point.

It has to be emphasized, that even though the geometry visually resembles the homoclinic tangle in 2D symplectic maps, the iterate of any of the homoclinic intersections in general is not contained in the $3 \mathrm{D}$ phase-space slice. Actually, we find numerically that the stable and unstable manifolds intersect in a 1D line which is itself an invariant set. Therefore, the intersection point in the 3D phase-space slice and its iterates are only a subset of the 1D intersection line. Moreover, as the manifolds are only 2D they cannot enclose a volume, so that there is no equivalent to the lobe structure and transport via a turnstile mechanism as in 2D symplectic maps [87 90]. 


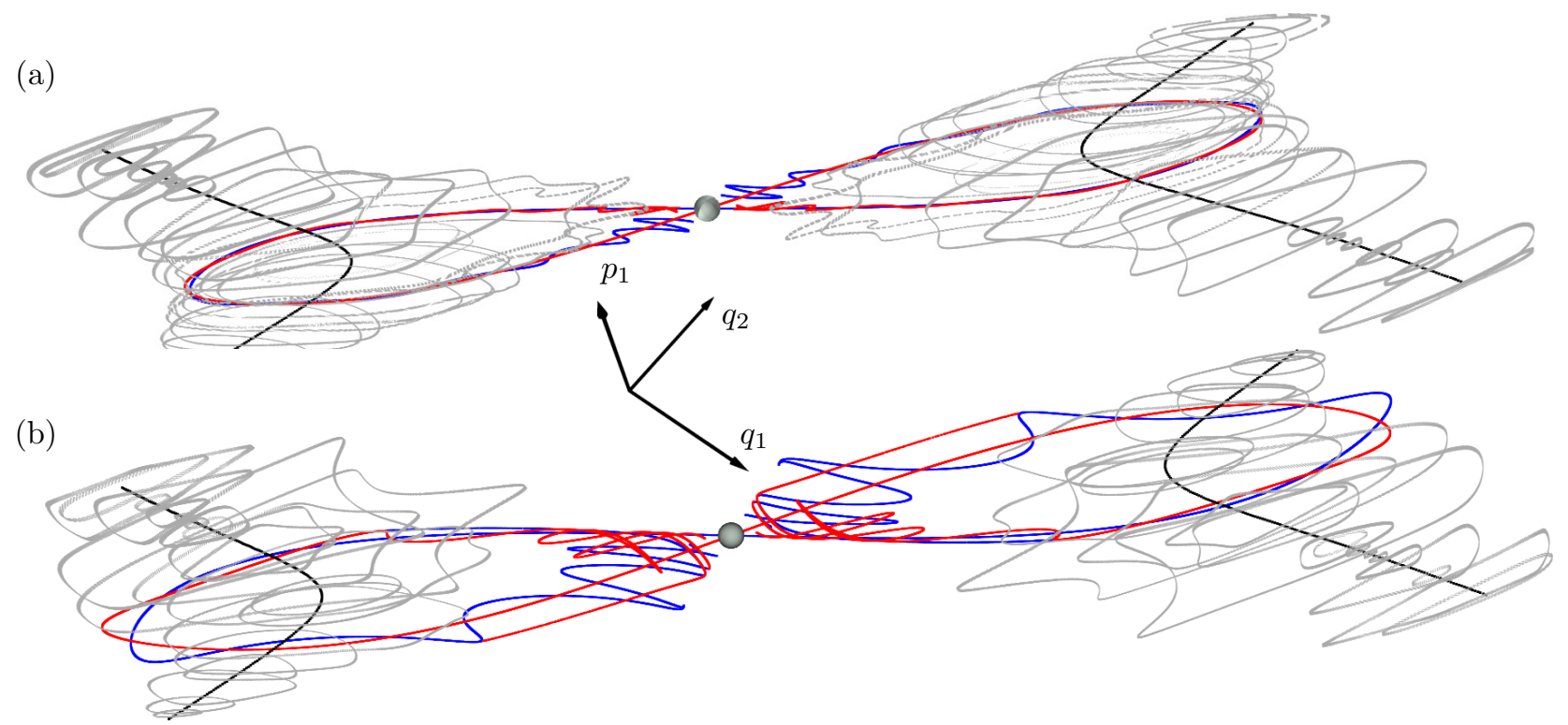

FIG. 10. 3D phase-space slice representation of the stable (blue) and unstable (red) manifolds of the CU fixed point together with regular 2-tori (gray) and the family of 1 D tori (black) for (a) $K_{1}=0.285$ and (b) $K_{1}=0.28$. Thus (a) corresponds to the point (F) in Fig. 4, compare with Fig. 6(c), (f). For a rotating view see http://www.comp-phys.tu-dresden.de/supp/

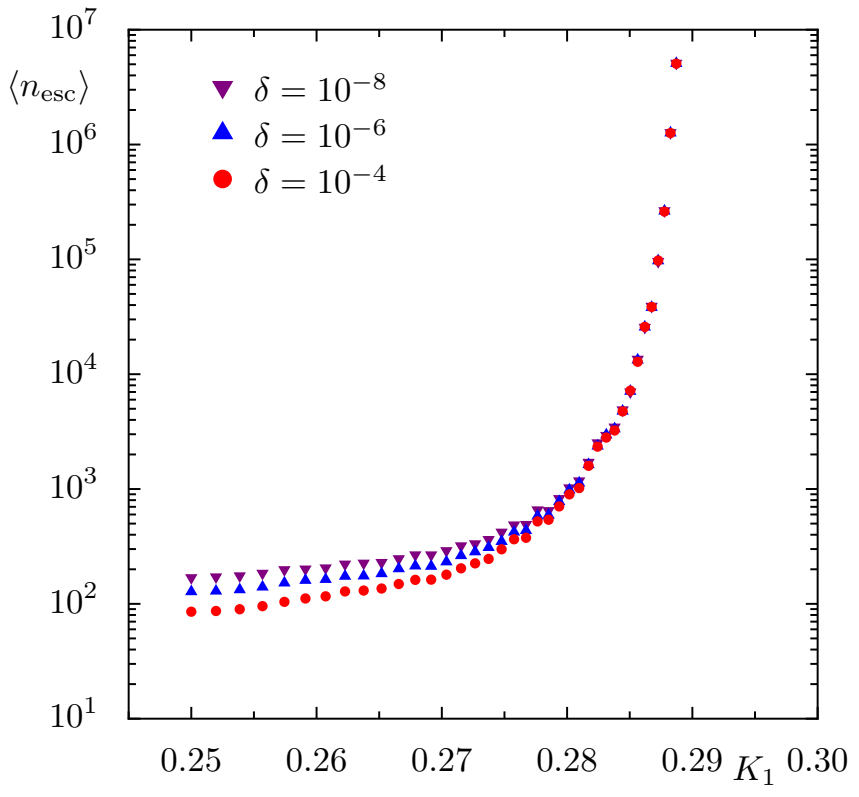

FIG. 11. Average escape time $\left\langle n_{\text {esc }}\right\rangle$ of an ensemble of $10^{4}$ orbits started in $U_{\delta}$ in dependence on $K_{1}$ for $\delta=10^{-8}$ (purple downward triangles), $\delta=10^{-6}$ (blue triangles), and $\delta=10^{-4}$ (red circles).

\section{Escape statistics}

To investigate the chaotic transport in the vicinity of the $\mathrm{CU}$ fixed point we consider an ensemble of initial conditions in a $4 \mathrm{D}$ cube

$$
U_{\delta}=[-\delta, \delta]^{2} \times[0.5-\delta, 0.5+\delta]^{2},
$$

with small $\delta$. The exit region is again chosen to be $p_{1}, p_{2} \in[-0.5,0.5]$ and $q_{1}, q_{2} \notin[0.25,0.75]$. Figure 11 shows the average escape time $\left\langle n_{\text {esc }}\right\rangle$ for an ensemble of $10^{4}$ orbits in dependence on $K_{1}$ for different $\delta=10^{-4}$, $\delta=10^{-6}$, and $\delta=10^{-8}$. When approaching the Krein collision parameter $K_{1}^{*}=0.3$, the average escape time $\left\langle n_{\text {esc }}\right\rangle$ strongly increases and for $K_{1}>0.29$ exceeds $10^{7}$ iterations. The same is also found for the smallest escape time (not shown). Extracting the functional dependence from the data turned out to inconclusive.

The tail of the distribution $P\left(n_{\text {esc }}\right)$ of escape times is very well described by an exponential, see Fig. 12, This provides a hint at what mechanism could be responsible for such large escape times: there could be one partial barrier (of unknown origin) for the dynamics which allows for a small flux towards the escape region [89. Such a a single partial barrier would lead to a simple exponential 91 while in contrast several partial barriers would typically lead to an overall power-law behavior 9294 . Note that for the small hump of $\left\langle n_{\text {esc }}\right\rangle$ seen in Fig. 11 around $K_{1}=0.284$ the corresponding $P\left(n_{\text {esc }}\right)$ shows a non-exponential behavior in the tail.

To quantify the escape dynamics of the ensemble, we now consider the extent as a function of the number of iterates. Explicitly we determine

$$
d_{\max }(n)=\max _{i \leq n}\left\{\left\|\boldsymbol{z}^{(i)}-\boldsymbol{z}^{*}\right\| \mid \text { with } \boldsymbol{z}^{(0)} \in U_{\delta}\right\},
$$

where $\boldsymbol{z}^{(i)}$ is the $i$-th iterate of an initial point $\boldsymbol{z}^{(0)} \in U_{\delta}$ and $\left\|\boldsymbol{z}_{i}-\boldsymbol{z}^{*}\right\|$ is the distance to the complex unstable fixed point at $\boldsymbol{z}^{*}$. We use $10^{4}$ initial conditions in $U_{\delta}$ with $\delta=10^{-6}$. Figure 13 shows the result for five different values of $K_{1}$. The expansion during the first 100 iterations is similar and after about 10 iterations follows 


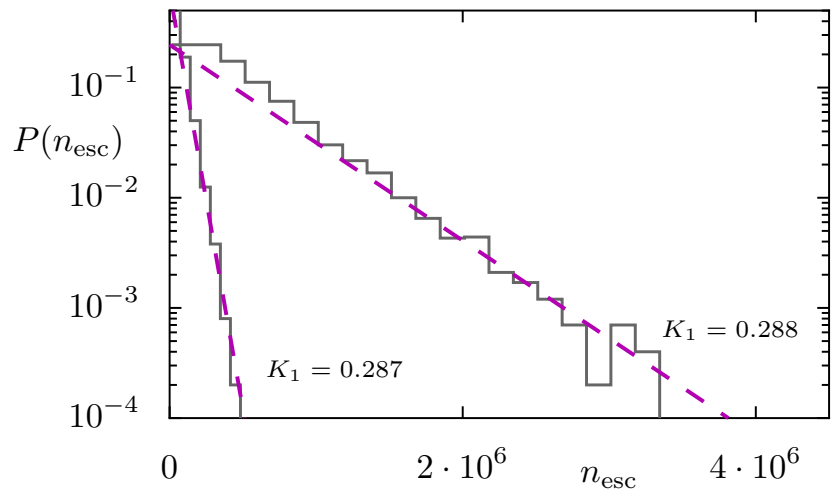

FIG. 12. Histogram $P\left(n_{\text {esc }}\right)$ of the escape times for $K_{1}=$ 0.287 and $K_{1}=0.288$. The dashed lines show a fit to an exponential for large $n_{\mathrm{esc}}$

an overall exponential given by $|\lambda|^{n}$, where $\lambda$ is the eigenvalue with largest absolute value. For $K_{1}=0.28$ this is illustrated by the blue dashed curve. On a finer scale the initial expansion happens in a step-like manner. This is due to the spiraling motion of each orbit as illustrated in Fig. 5. This motion has a different extent in the different directions, so that a larger distance is only obtained periodically after approximately ten iterations for the first expansion phase. This corresponds to half the reciprocal winding frequency of the fixed point.

After the first rapid expansion phase, the maximal distance shows prominent plateaus extending over several

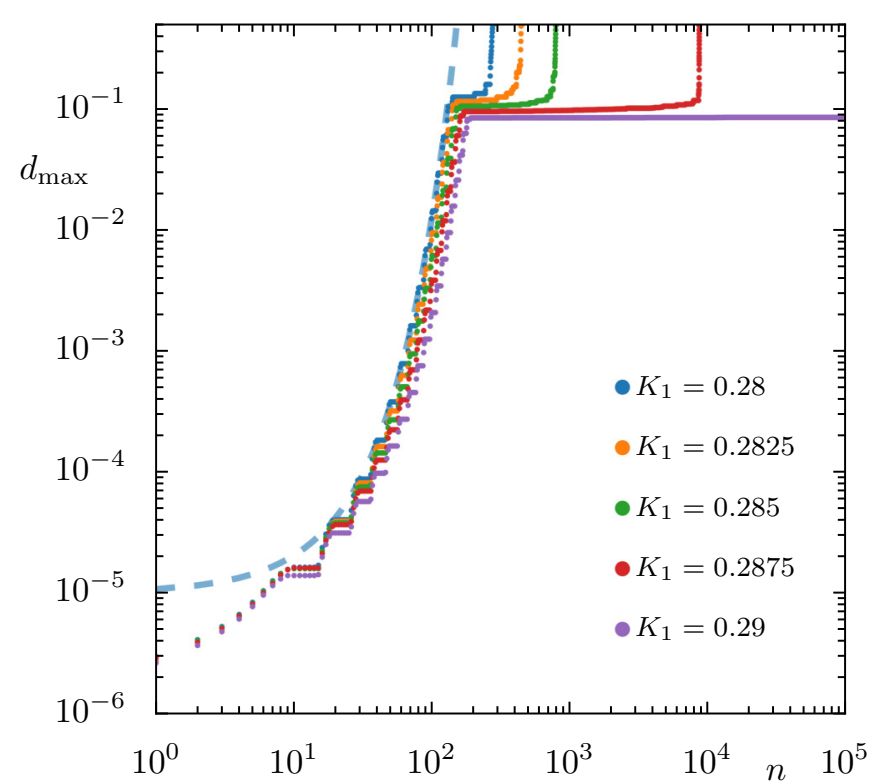

FIG. 13. Maximal distance $d_{\max }$ of an ensemble of $10^{4}$ initial conditions started in the $4 \mathrm{D}$ cube $U_{\delta}$ with $\delta=10^{-6}$ vs. the number of iterations $n$ for $K_{1}=$ $0.28,0.2825,0.285,0.2875,0.29$ (top to bottom, corresponding to increasing escape time). The initial expansion is well described by $\propto|\lambda|^{n}$, shown for $K_{1}=0.28$ (blue dashed curve). orders of magnitude in time. These plateaus become longer the closer the parameter $K_{1}$ is to $K_{1}^{*}=0.3$, i.e. the parameter of the Krein collision. Thus for a very long time the ensemble is effectively confined in phase space. Afterwards there is at least one trajectory which leaves this region very quickly, as manifested by the sharp increase of $d_{\max }$.

A closer look at the plateaus reveals that there is still a rather slow increase. The occurrence of the plateaus can be explained by the alternating spiraling in and out of the dynamics already observed in Refs. 6, 11, 36, 38: An orbit initially started near the complex unstable fixed point moves away from it on a spiral along the unstable manifold until it reaches a maximal distance to the fixed point. This behavior corresponds to the first expansion phase up to approximately 100 iterations. Subsequently, the orbit spirals in again and gets very close to the fixed point with some minimal distance. When spiraling out again, this can lead to a slightly increased maximal distance. This process of inward and outward spiraling repeats many times before the orbit escapes quickly. Note, that this sequence of outward and inward spiraling only holds for parameters which are near the elliptic-elliptic region in the parameter plot in Fig. 4, i.e. if $K_{1}$ is sufficiently close to $K_{1}^{*}=0.3$. Further away from the Krein collision parameter the extent of the plateau of $d_{\max }$ becomes very short or even non-existent, see Fig. 13 for $K_{1}=0.28$.

It is also illuminating to consider the extent of the iterated ensemble at a given number of iterations,

$$
\widetilde{d}_{\max }(n)=\max \left\{\left\|\boldsymbol{z}^{(n)}-\boldsymbol{z}^{*}\right\| \mid \text { with } \boldsymbol{z}^{(0)} \in U_{\delta}\right\},
$$

see Fig. 14. Initially one has the overall exponential increase which is superimposed by small oscillation caused by the spiraling motion. This occurs until the ensemble has expanded until the homoclinic intersection, which corresponds to the beginning of the plateaus in Fig. 13. Afterwards, there is a prominent dip around $n=200$ i.e. the extent of the ensemble has become quite small again and most of the points are located in a small surrounding of the complex unstable fixed point. These minima converge to the plateau for growing $n$ such that the second dip is already barely visible. This effect is due to the inward and outward spiraling behavior of each individual orbit. The inset of Fig. 14 shows $\widetilde{d}_{\max }$ of one single orbit. The position of the first minimum after the expansion of one single orbit matches roughly the first minimum in the plateau of the ensemble. This expansion and contraction of the ensemble repeats approximately periodically until some loss of correlations sets in and the dips of $d_{\max }$ become less and less prominent. Note that such kind of dynamics is also found for 2D symplectic maps for the dynamics after a period-doubling bifurcation and also for 4 D symplectic maps with an II fixed-point. A more detailed investigation and comparison of these cases would be very interesting and is left for future studies. 


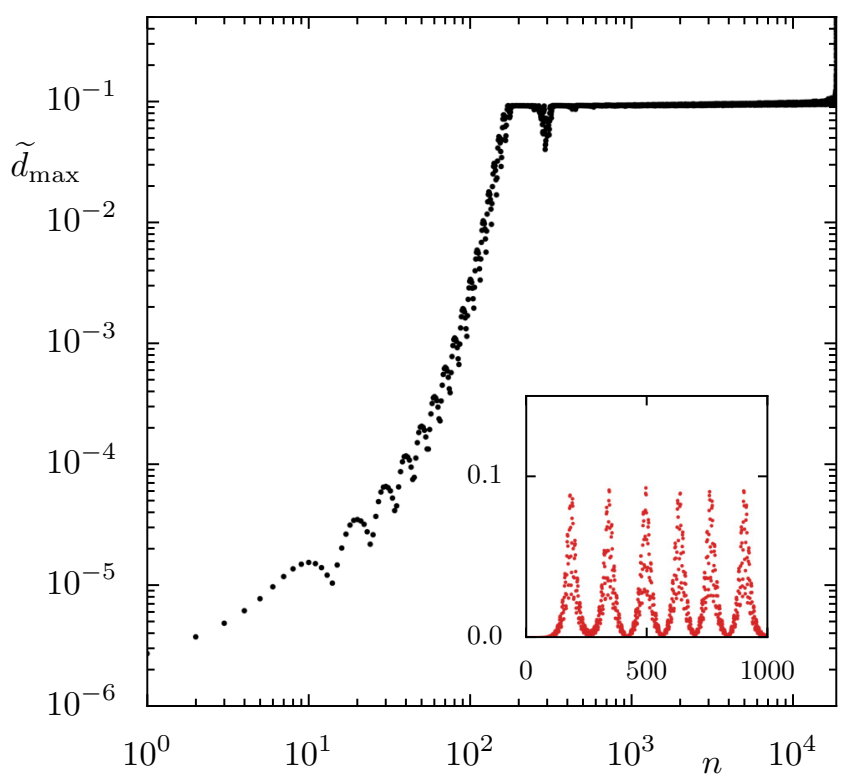

FIG. 14. Maximal extent $\widetilde{d}_{\text {max }}$ of an ensemble of $10^{4}$ initial conditions started in the $4 \mathrm{D}$ cube $U_{\delta}$ with $\delta=10^{-6}$ vs. the number of iterations $n$. The inset shows the maximal extent of a single exemplary orbit up to the first 1000 iterations.

\section{Escape dynamics}

The temporal dependence of the extent of the iterates of the ensemble allows for quantifying the long-time confinement within the chaotic region surrounding the complex unstable fixed point. Still, the key question is, what is responsible for this long-time confinement and what is the escape mechanism? In particular, referring to the normal form description, there could be either an escape within the $I=0$ plane or across different planes with $I \neq 0$. Escape within $I=0$ would be similar to the case of the period-doubling bifurcation in 2D symplectic maps, where just after the fixed point has become unstable there are usually still invariant curves so that an escape of orbits is only possible when being further away from the bifurcation in parameter space. In contrast, the escape across different planes with $I \neq 0$ would be a genuinely $4 \mathrm{D}$ effect. In principle there could also be a competition between these two escape routes and which of them is relevant could depend both on parameters and considered time-scales.

As a measure of the invariant $I$ of the normal form for a symplectic map we make use of the quadratic invariant of the linearized map. With Eq. (6) we get

$$
\begin{aligned}
Q= & -p_{1}^{2}+p_{2}^{2}-q_{1}^{2}\left(K_{1}-K\right)-q_{2}^{2}\left(K_{2}-K\right) \\
& +p_{1} q_{1}\left(K-K_{1}\right)+p_{2} q_{2}\left(K_{2}-K\right) \\
& +K\left(p_{1} q_{2}-p_{2} q_{1}+2 q_{1} q_{2}\right) .
\end{aligned}
$$

By use of a suitable coordinate transformation Eq. 29 degenerates for the Krein collision parameter into two planes, namely the $p_{1}=-p_{2}$ and the $q_{1}=q_{2}$ plane 33 . (a)
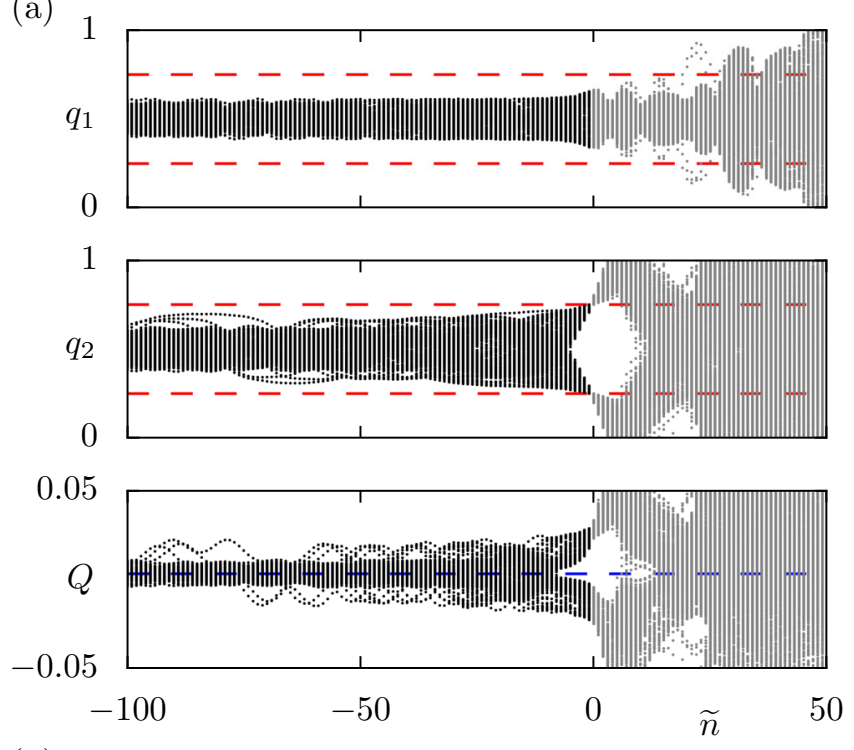

(b)

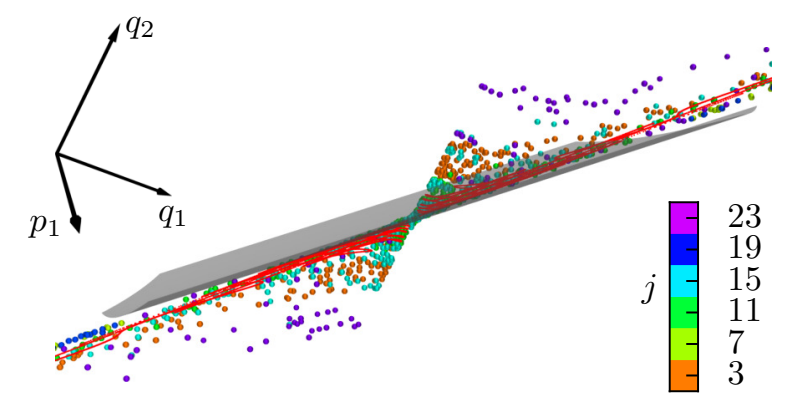

FIG. 15. (a) Shown are the $q_{1}, q_{2}$-coordinates and the quadratic invariant of the linearization $Q$ of 1000 orbits started with random initial conditions in $U_{\delta}$ with $\delta=10^{-6}$ and $K_{1}=0.288$ over $\tilde{n}=n-n_{\text {esc }}$. The escape criterion is the same as in the previous experiments and marked as a red dashed line while in the last plot the blue dashed line represents $Q_{0}$. (b) 3D phase-space slice representation of segments of a single exemplary orbit for $K_{1}=0.288$. Each segment consists of consecutive 10000 iterates and shown are those points fulfilling the slice condition for the segments $j=3,7,11,15,19$, and 23 , see the text for further explanation. The unstable manifold is shown as a red curve and the $Q=Q_{0}$ plane as gray transparent surface. For a rotating view see http://www.comp-phys.tu-dresden.de/supp/

These two planes geometrically correspond to the representation of the $I=0$ plane for the hyperplanes $x_{2}=0$ or alternatively $y_{2}=0$ in the normal form description, see section IIC. Hence, the quadratic invariant at the fixed point is $Q\left(\boldsymbol{z}^{*}\right)=0$.

However, away from the Krein collision the two planes are not degenerate anymore. Therefore $Q$ does not resemble the $I=0$ plane and we get

$$
Q_{0}=Q\left(z^{*}\right)=-\frac{K_{1}+K_{2}}{4}+K
$$

which is not zero in general. Still it turns out, that $Q-Q_{0}$ is a well suited quantity to approximate the invariant $I$ 
of the normal form for a symplectic map.

To address the question of the possible escape route, it is helpful to compare for an ensemble of initial conditions the individual coordinates of the orbits right before they escape. Figure 15(a) shows the $q_{1}$ and $q_{2}$ coordinates as well as the quadratic invariant $Q$ as function of $\widetilde{n}=n-n_{\text {esc }}$, i.e. for a few iterations before and after the escape of an orbit. The initial conditions of the ensemble with 1000 orbits are started in $U_{\delta}$ with $\delta=10^{-6}$ and the kicking strength is $K_{1}=0.288$. The orbits are confined for negative $\widetilde{n}$ and fulfill the escape criterion for positive $\widetilde{n}$, as indicated by the red dashed horizontal lines. The spread of the distances of $q_{1}$ and $q_{2}$ around the fixed point, i.e. the width of the distribution of distances around 0.5 , is slightly increasing towards $\tilde{n}=0$. Even though this trend is visible in both coordinates, the escape condition is reached first by the $q_{2}$ coordinate.

In order to understand the escape mechanism in terms of the phase space geometry, we compare the escape path in the 3D phase-space slice with the geometry of the normal-form. The arrangement of regular tori and the family of 1D tori, see Fig. 6, suggest that the $Q=Q_{0}$ plane is a good approximation to the $I=0$ plane, compare to the gray plane in Fig. 15(b). Therefore, $Q-Q_{0}$ provides an approximate measure of how far a point of an orbit is away from the $I=0$ plane, see Fig. 15(a). As for the single coordinates, $Q$ shows an overall increase and is spread more widely as $\widetilde{n}$ approaches 0 . However, about 7 iterations before $\widetilde{n}=0$ the distribution of $Q$ splits into two separate parts, away from 0 .

In order to determine if the ensemble escapes through these two separated escape paths or interchanges between those two, we split the ensemble in two subsets by either $Q(\tilde{n}=0)>Q_{0}$ or $Q(\widetilde{n}=0)<Q_{0}$ and determine their mean and variance. Figure 16.(a) shows the average as dots and their standard deviation as error bars of the $Q(\tilde{n}=0)>Q_{0}$ and the $Q(\tilde{n}=0)<Q_{0}$ subset in blue and red color, respectively. The ensemble clearly separates in these two sets and fluctuates around $Q_{0}$ marked as a black dashed line. Once the escape criterion is fulfilled, either $Q>Q_{0}$ or $Q<Q_{0}$ and initially no further change in sign occurs. This behavior translates to escape either across $I>0$ or $I<0$ planes in the normalform picture. Crossing the planes with different $I$ is only possible because the normal-form geometry provided by Eq. 18 is broken.

Figure 16.(b) shows the time evolution of the variance of both sets ranging from 2000 iterations before the escape up to the escape. We observe the same type of increase of the variance for both subsets towards the escape at $\tilde{n}=0$. Understanding the behaviour of the variance quantitatively is an interesting future task.

By following one single orbit we can also get an intuition of how the orbit crosses the different $I \neq 0$ planes, see Fig. 15(b). Here, we consider a single orbit with initial condition $\left(p_{1}, p_{2}, q_{1}, q_{2}\right)=(0,0,0.5+\mu, 0.5+\mu)$ with $\mu=10^{-5}$ for $K_{1}=0.288$. This orbit escapes after approximately 266000 iterations in our numerical imple-
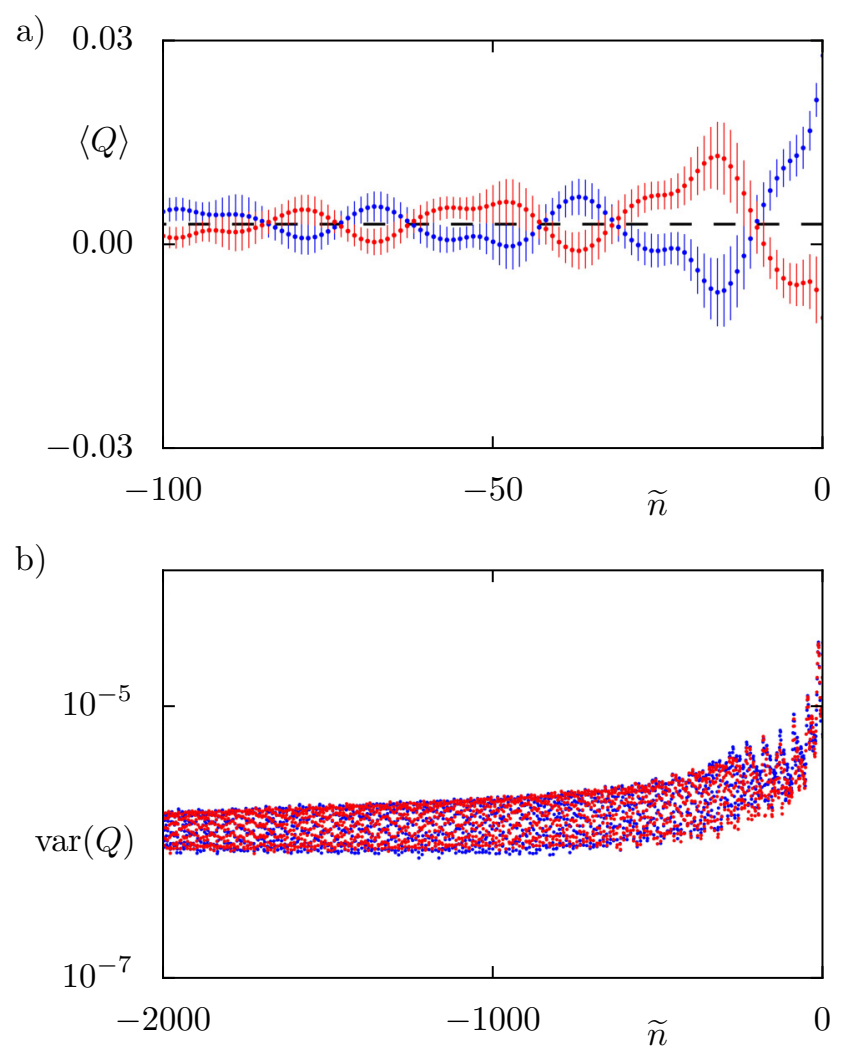

FIG. 16. The mean width standard deviation a) and the variance b) of the quadratic invariants $Q$ of the ensemble in Fig. 15 are shown. The blue set corresponds to the set of orbits with $Q(\widetilde{n}=0)>Q_{0}$ and the inverse to the red data points. The black dashed line in a) represents $Q_{0}$

mentation of the map. For this orbit we consider successive segments $[j \cdot 10000,(j+1) \cdot 10000]$ of the iterates of the orbit. For each segment those points fulfilling the slice condition (23) with $\varepsilon=10^{-4}$ are determined. A selection in the surrounding of the complex unstable fixed point is shown in Fig. 15(b) together with the 3D phase-space slice of the unstable manifold as a red curve. This plot shows that the iterates of the initial point are approximately restricted around $1 \mathrm{D}$ lines in the 3D phase-space slice. These lines follow the unstable manifold and each of the successive segments appears to lie on a slightly bent surface, similar to the $I \neq 0$ planes of the normal form, compare with Fig. 3. This suggests that an escaping orbit is following the unstable manifold which gives rise to transport through the $I \neq 0$ planes. Note that the slice segments for $j=7,19$ are located at the excursion of the manifold farther away from the fixed point and therefore do only appear at the edge of the magnification. In general, the motion along the unstable manifold explains also the repetitive expanding and contracting behavior of the orbits. 


\section{SUMMARY AND OUTLOOK}

In this paper the transition of a fixed point with elliptic-elliptic dynamics to complex-unstable dynamics under parameter variation is investigated for a 4D symplectic map. Using 3D phase-space slices we visualize regular dynamics in the vicinity of the fixed point. While in the elliptic-elliptic case there exist two families of $1 \mathrm{D}$ tori which are attached to the fixed point and are surrounded by regular 2-tori, these families merge into one single family and split off the fixed point. Moreover, the geometry of regular orbits close to the fixed point in the 3D phase-space slice lie on surfaces as predicted by the normal form description, see Fig. 3. The phase-space representation is complemented by a frequency analysis of regular tori, see Fig. 8. Before the transition to complex instability the two families of 1D tori are attached to the fixed point forming a cusp-like region which encloses the regular tori. The fixed point becomes complex unstable under parameter variation when reaching the $-1: 1: 0$ resonance line and the families of $1 \mathrm{D}$ tori split off the fixed point. Applying a unimodular transformation clarifies that these apparently two families of 1D tori actually form a single arc in frequency space.

Once the fixed point has become complex unstable nearby orbits may eventually escape. However, it turns out that shortly after the transition orbits are confined to a particular phase-space region for very long times. This region can be visualized using escape time plots, see Fig. 9. The extent is governed by the stable and unstable invariant manifolds of the complex unstable fixed point. In the 3D phase space they lead to a geometry which is visually similar to that of the well-known homoclinic tangle for 2D symplectic maps.

To quantify these observations we consider the escape statistics for an ensemble of $10^{4}$ orbits, started in the vicinity of the fixed point in dependence on the distance to the bifurcation point, i.e. by varying the parameter $K_{1}$. The average escape time strongly increases when approaching the bifurcation point. Measuring the maximal distance of all orbits of the ensemble to the fixed point over the number of iterations, reveals three different phases of the dynamics, see Fig. 13. Initially, for the first approximately 100-200 iterations, the distance increases exponentially, followed by a extended plateaus in the second phase. These plateaus correspond to the longtime confinement and extend over longer times the closer the parameter is to the Krein bifurcation. A closer look at the plateaus shows that there is a very slow increase as function of time. The plateaus are due to the inward and outward spiraling dynamics of the ensemble which follows the unstable invariant manifold. Thus, the slope corresponds to a slowly growing extent of individual orbits, see Fig. 14. Eventually, in the last phase one orbit of the ensemble will escape after a critical time and the maximal distance of the ensemble quickly reaches approximately 1 . If the fixed point is very unstable, the plateau is very short or even not existent.
Comparing the $q_{1}, q_{2}$ coordinates and the quadratic invariant $Q$ of the ensemble for the transition from phase two to three allows for determining the main escape paths close to the bifurcation, see Fig. 15. This provides evidence that long confined orbits escape across either $I>0$ or $I<0$ planes of the normal-form. Thus the escape mechanism is genuinely higher-dimensional.

Based on the improved understanding of the geometry and escape of orbits near a complex unstable fixed point, an interesting future task is to explicitly determine the invariant $I$ for the specific map using a numerical normal form analysis. This would allow for accurately quantifying the transport across the approximately invariant planes and to investigate whether the escape can be described by a diffusive process.

\section{ACKNOWLEDGMENTS}

We are grateful for discussions with Markus Firmbach, Franziska Hübner, Roland Ketzmerick, and Haris Skokos. Robert MacKay kindly provided us with a copy of Ref. [40]. Furthermore, we acknowledge support by the Deutsche Forschungsgemeinschaft under grant KE 537/6-1.

All 3D visualizations were created using MAYAVI [95].

\section{Appendix A: Computing stable and unstable manifolds}

There are various methods to determine the invariant manifolds associated with an unstable fixed point, see e.g. Refs. 96 101. Here we use the parametrization method, which was introduced in Refs. 48, 50, and has been used for example in Refs. [77, 85, 86].

The parameterization method takes advantage of the Hartman-Grobman theorem which for symplectic maps states that the linearization of a fixed point or periodic orbit is conjugate to its local stable and unstable invariant manifolds $W_{\mathrm{loc}}^{\mathrm{s}, \mathrm{u}}$, if the eigenvalues have an absolute value different from one, i.e., if they are unstable. The key point of the parameterization method is to find smooth vector-valued functions $\mathcal{F}_{\mathrm{s}}$ and $\mathcal{F}_{\mathrm{u}}$ which parameterize the stable and unstable invariant manifolds. In order to do so, $\mathcal{F}_{\mathrm{s}, \mathrm{u}}$ have to obey on the one hand the linear conditions

$$
\begin{aligned}
\mathcal{F}_{s, u}(\mathbf{0}) & =\boldsymbol{z}^{*} \quad \text { and } \\
\frac{\partial \mathcal{F}_{s, u}(\boldsymbol{\theta})}{\partial \theta_{j}} & =\boldsymbol{\xi}_{j} \quad \text { for } \quad 1 \leq i \leq n_{s, u}
\end{aligned}
$$

with $\boldsymbol{\theta}=\left(\theta_{1}, \ldots, \theta_{n_{s, u}}\right) \in \mathbb{C}^{n_{s, u}}$ and $\xi_{j} \in \mathbb{C}^{2 n_{s, u}}$ being the associated eigenvector to the $n_{s}$ stable and $n_{u}$ unstable eigenvalues $\lambda_{j}$.

On the other hand, $\mathcal{F}_{s, u}$ must satisfy the conjugacy equation

$$
\mathcal{M} \circ \mathcal{F}_{s, u}(\boldsymbol{\theta})=\mathcal{F}_{s, u}\left(\lambda_{1} \theta_{1}, \ldots, \lambda_{n_{s, u}} \theta_{n_{s, u}}\right)
$$


in order to take the non-linearity of the map into account.

For a complex unstable fixed point of a 4D symeplectic map one finds $n_{s}=n_{u}=2$. Therefore, we expand $\mathcal{F}_{s, u}$ into the power series

$$
\mathcal{F}_{s, u}\left(\theta_{1}, \theta_{2}\right)=\left(\begin{array}{c}
p_{1}\left(\theta_{1}, \theta_{2}\right) \\
p_{2}\left(\theta_{1}, \theta_{2}\right) \\
q_{1}\left(\theta_{1}, \theta_{2}\right) \\
q_{2}\left(\theta_{1}, \theta_{2}\right)
\end{array}\right)=\sum_{i=0}^{\infty} \sum_{j=0}^{\infty} \boldsymbol{f}_{i j} \theta_{1}^{i} \theta_{2}^{j},
$$

with vector-valued coefficients $\boldsymbol{f}_{i j} \in \mathbb{C}^{4}$.

For the considered map (18), the non-linear terms consist of sine functions with various input arguments, namely three different sums of phase-space coordinates. We approximate these sine functions by their Taylor series representation. Advantageously, the coefficients of this series can be easily computed by an autodifferentiation algorithm which is based on Refs. [102, 103. Using the series representation of the sine terms of the map $\mathcal{M}$ and combining $(\mathrm{A} 4$ and the conjugacy equation A3 leads to a homological equation which can be solved iteratively for the coefficients $\boldsymbol{f}_{i j}$ up to a given order $(m, n)$. The corresponding initial value problem is solved by the linear conditions (A1) and (A2).
[1] R. Broucke, Stability of periodic orbits in the elliptic, restricted three-body problem., AIAA Journal 7, 1003 (1969).

[2] J. D. Hadjidemetriou, The stability of periodic orbits in the three-body problem, Celestial Mech. 12, 255 (1975).

[3] B. Sicardy, Stability of the triangular Lagrange points beyond Gascheau's value, Celest. Mech. Dyn. Astron. 107, 145 (2010).

[4] P. Magnenat, Numerical study of periodic orbit properties in a dynamical system with three degrees of freedom, Celestial Mech. 28, 319 (1982).

[5] L. Martinet and D. Pfenniger, Complex instability around the rotation axis of stellar systems. I. Galactic potentials, Astron. \& Astrophys. 173, 81 (1987).

[6] D. C. Heggie, Bifurcation at complex instability, Celestial Mech. 35, 357 (1985).

[7] G. Contopoulos and P. Magnenat, Simple threedimensional periodic orbits in a galactic-type potential, Celestial Mech. 37, 387 (1985).

[8] P. A. Patsis and L. Zachilas, Complex instability of simple periodic orbits in a realistic two-component galactic potential, Astron. \& Astrophys. 227, 37 (1990).

[9] M. Ollé, J. R. Pacha, and J. Villanueva, Motion close to the hopf bifurcation of the vertical family of periodic orbits of 14 , Celest. Mech. Dyn. Astron. 90, 87 (2004).

[10] H. Hanßmann and J.-C. van der Meer, Algebraic methods for determining Hamiltonian Hopf bifurcations in three-degree-of-freedom systems, J. Dyn. Diff. Equat. 17, 455 (2005).

[11] M. Katsanikas, P. A. Patsis, and G. Contopoulos, The structure and evolution of confined tori near a Hamiltonian Hopf bifurcation, Int. J. Bifurcation Chaos 21, 2321 (2011).

[12] P. A. Patsis and M. Katsanikas, The phase space of boxy-peanut and X-shaped bulges in galaxies - II. The relation between face-on and edge-on boxiness, Mon. Not. R. Astron. Soc. 445, 3546 (2014).

[13] K. Efstathiou, R. H. Cushman, and D. A. Sadovskií, Hamiltonian Hopf bifurcation of the hydrogen atom in crossed fields, Physica D 194, 250 (2004).

[14] A. Lahiri and M. S. Roy, The Hamiltonian Hopf bifurcation, International Journal of Non-Linear Mechanics 36, 787 (2001).

[15] M. Ollé and J. R. Pacha, Hopf bifurcation for the hydrogen atom in a circularly polarized microwave field, Commun. Nonlinear Sci. Numer. Simulat. 62, 27 (2018).
[16] S. C. Farantos and M. Founargiotakis, Wave packet dynamics and phase space structure of HCN molecule, Chemical Physics 142, 345 (1990).

[17] G. Díaz, J. Egea, S. Ferrer, J. C. van der Meer, and J. A. Vera, Relative equilibria and bifurcations in the generalized van der Waals 4 D oscillator, Physica D 239, 1610 (2010).

[18] J. E. Howard, A. J. Lichtenberg, M. A. Lieberman, and R. H. Cohen, Four-dimensional mapping model for twofrequency electron cyclotron resonance heating, Physica D 20, 259 (1986).

[19] J.-C. van der Meer, The Hamiltonian Hopf Bifurcation, number 1160 in Lecture Notes in Mathematics, Springer Berlin Heidelberg (1985).

[20] J. D. Crawford, Introduction to bifurcation theory, Rev. Mod. Phys. 63, 991 (1991).

[21] H. Papadaki, G. Contopoulos, and C. Polymilis, Complex instability, in A. E. Roy and B. A. Steves (editors) "From Newton to Chaos", 485, Plenum Press, New York (1995).

[22] P. D. McSwiggen and K. R. Meyer, The evolution of invariant manifolds in Hamiltonian-Hopf bifurcations, J. Diff. Eqs. 189, 538 (2003).

[23] M. Ollé, J. R. Pacha, and J. Villanueva, Dynamics and bifurcation near the transition from stability to complex instability, in J. Delgado, E. A. Lacomba, J. Llibre, and E. Pérez-Chavela (editors) "New Advances in Celestial Mechanics and Hamiltonian Systems", 185, Springer US (2004).

[24] M. Ollé, J. R. Pacha, and J. Villanueva, Dynamics close to a non semi-simple 1:-1 resonant periodic orbit, Discrete Contin. Dyn. Sys. Ser. B 5, 799 (2005).

[25] E. Fontich, C. Simó, and A. Vieiro, Splitting of the separatrices after a Hamiltonian-Hopf bifurcation under periodic forcing, Nonlinearity 32, 1440 (2019).

[26] G. Wen, Criterion to identify Hopf bifurcations in maps of arbitrary dimension, Phys. Rev. E 72, 026201 (2005).

[27] H. W. Broer, H. Hanßmann, and J. Hoo, The quasiperiodic Hamiltonian Hopf bifurcation, Nonlinearity 20, 417 (2007).

[28] M. Ollé, J. R. Pacha, and J. Villanueva, KolmogorovArnold-Moser aspects of the periodic hamiltonian Hopf bifurcation, Nonlinearity 21, 1759 (2008).

[29] R. Vitolo, H. Broer, and C. Simó, Quasi-periodic bifurcations of invariant circles in low-dimensional dissipa- 
tive dynamical systems, Regul. Chaotic Dyn. 16, 154 (2011).

[30] G. Contopoulos, S. C. Farantos, H. Papadaki, and C. Polymilis, Complex unstable periodic orbits and their manifestation in classical and quantum dynamics, Phys. Rev. E 50, 4399 (1994).

[31] J. E. Howard and R. S. MacKay, Linear stability of symplectic maps, J. Math. Phys. 28, 1036 (1987).

[32] Ch. Skokos, On the stability of periodic orbits of high dimensional autonomous Hamiltonian systems, Physica D 159, 155 (2001).

[33] D. Pfenniger, Numerical study of complex instability. I. Mappings, Astron. \& Astrophys. 150, 97 (1985).

[34] D. Pfenniger, Numerical study of complex instability. II. barred galaxy bulges, Astron. \& Astrophys. 150, 112 (1985).

[35] C. Froeschle, On the number of isolating integrals in systems with three degrees of freedom, Astrophys. Space Sci. 14, 110 (1971).

[36] À. Jorba and M. Ollé, Invariant curves near Hamiltonian-Hopf bifurcations of four-dimensional symplectic maps, Nonlinearity 17, 691 (2004).

[37] L. Zachilas, M. Katsanikas, and P. A. Patsis, The structure of phase space close to fixed points in a $4 \mathrm{D}$ symplectic map, Int. J. Bifurcation Chaos 23, 1330023 (2013).

[38] N. Delis and G. Contopoulos, Analytical and numerical manifolds in a symplectic 4-D map, Celest. Mech. Dyn. Astron. 126, 313337 (2016).

[39] T. K. Roy and A. Lahiri, Reversible Hopf bifurcation in four-dimensional maps, Phys. Rev. A 44, 4937 (1991).

[40] T. J. Bridges, Cushman, R. H., and R. S. MacKay, Dynamics near an irrational collision of eigenvalues for symplectic maps, Fields Institute Communications 4, 61 (1995).

[41] E. Fontich, C. Simó, and A. Vieiro, The discrete Hamiltonian-Hopf bifurcation for $4 D$ symplectic maps, in M. Corbera, J. M. Cors, J. Llibre, and A. Korobeinikov (editors) "Extended Abstracts Spring 2014", Number 4 in Trends in Mathematics, 77, Springer International Publishing Switzerland (2015).

[42] A. Lahiri, A. Bhowal, and T. K. Roy, Resonant collisions in four-dimensional reversible maps: A description of scenarios, Physica D 112, 95 (1998).

[43] A. Bhowal, T. K. Roy, and A. Lahiri, Hopf bifurcation in four-dimensional reversible maps and renormalisation equations, Phys. Lett. A 179, 9 (1993).

[44] M. Richter, S. Lange, A. Bäcker, and R. Ketzmerick, Visualization and comparison of classical structures and quantum states of four-dimensional maps, Phys. Rev. E 89, 022902 (2014).

[45] J. Laskar, The chaotic motion of the solar system: A numerical estimate of the size of the chaotic zones, Icarus 88, 266 (1990).

[46] J. Laskar, Frequency analysis for multi-dimensional systems. Global dynamics and diffusion, Physica D 67, 257 (1993).

[47] J. Laskar, Frequency analysis of a dynamical system, Celest. Mech. Dyn. Astron. 56, 191 (1993).

[48] X. Cabré, E. Fontich, and R. de la Llave, The parameterization method for invariant manifolds I: Manifolds associated to non-resonant subspaces, Indiana Univ. Math. J. 52, 283 (2003).
[49] X. Cabré, E. Fontich, and R. de la Llave, The parameterization method for invariant manifolds II: Regularity with respect to parameters, Indiana Univ. Math. J. 52, 329 (2003).

[50] X. Cabré, E. Fontich, and R. de la Llave, The parameterization method for invariant manifolds III, J. Diff. Eqs. 218, 444 (2005).

[51] J. Moser, New aspects in the theory of stability of Hamiltonian systems, Comm. Pure Appl. Math. 11, 81 (1958).

[52] V. I. Arnold and A. Avez, Ergodic Problems of Classical Mechanics, Benjamin, NewYork (1968).

[53] M. G. Kreı̆n, Topics in Differential and Integral Equations and Operator Theory, number 7 in Operator Theory: Advances and Applications (edited by I. Gohberg), Birkhäuser, Basel (1983).

[54] T. J. Bridges and R. H. Cushman, Unipotent normal forms for symplectic maps, Physica D 65, 211 (1993).

[55] T. J. Bridges and J. E. Furter, Singularity Theory and Equivariant Symplectic Maps, number 1558 in Lecture Notes in Mathematics, Springer Berlin Heidelberg (1993).

[56] K. Meyer and D. C. Offin, Introduction to Hamiltonian Dynamical Systems and the N-Body Problem, Springer International Publishing, Cham, third edition (2017).

[57] S. M. Graff, On the conservation of hyperbolic invariant tori for Hamiltonian systems, J. Diff. Eqs. 15, 1 (1974).

[58] E. Zehnder, Generalized implicit function theorems with applications to some small divisor problems, II, Comm. Pure Appl. Math. 29, 49 (1976).

[59] À. Jorba and J. Villanueva, On the normal behaviour of partially elliptic lower-dimensional tori of Hamiltonian systems, Nonlinearity 10, 783 (1997).

[60] À. Jorba and J. Villanueva, The fine geometry of the Cantor families of invariant tori in Hamiltonian systems, in C. Casacuberta, R. Miró-Roig, J. Verdera, and S. Xambó-Descamps (editors) "European Congress of Mathematics", volume 202 of Progress in Mathematics, 557, Birkhäuser Basel (2001).

[61] S. Lange, M. Richter, F. Onken, A. Bäcker, and R. Ketzmerick, Global structure of regular tori in a generic $4 D$ symplectic map, Chaos 24, 024409 (2014).

[62] F. Onken, S. Lange, R. Ketzmerick, and A. Bäcker, Bifurcations of families of $1 D$-tori in $4 D$ symplectic maps, Chaos 26, 063124 (2016).

[63] G. Contopoulos, Qualitative changes in 3-dimensional dynamical systems, Astron. \& Astrophys. 161, 244 (1986).

[64] G. Contopoulos and A. Giorgilli, Bifurcations and complex instability in a 4-dimensional symplectic mapping, Meccanica 23, 19 (1988).

[65] T. M. Cherry, On periodic solutions of Hamiltonian systems of differential equations, Phil. Trans. R. Soc. A 227, 137 (1928).

[66] K. R. Meyer, Generic bifurcation of periodic points, Trans. Amer. Math. Soc. 149, 95 (1970).

[67] J. M. Greene, R. S. MacKay, F. Vivaldi, and M. J. Feigenbaum, Universal behaviour in families of areapreserving maps, Physica D 3, 468 (1981).

[68] R. S. MacKay, Renormalisation in area-preserving maps, number 6 in Advanced Series in Nonlinear Dynamics, World Scientific, Singapure (1993). 
[69] K. Meyer, G. Hall, and D. Offin, Introduction to Hamiltonian Dynamical Systems and the N-Body Problem, Springer-Verlag, New York (2009).

[70] C. Froeschlé, Numerical study of dynamical systems with three degrees of freedom. I. Graphical displays of four-dimensional sections, Astron. \& Astrophys. 4, 115 (1970).

[71] C. Froeschlé, Numerical study of a four-dimensional mapping, Astron. \& Astrophys. 16, 172 (1972).

[72] À. Haro, Center and center-(un)stable manifolds of elliptic-hyperbolic fixed points of 4 d-symplectic maps. an example: The froeschlé map, in C. Simó (editor) "Hamiltonian Systems with Three or More Degrees of Freedom", volume 533 of NATO ASI Series: C - Mathematical and Physical Sciences, 403, Kluwer Academic Publishers, Dordrecht (1999).

[73] M. Guzzo, E. Lega, and C. Froeschlé, On the numerical detection of the effective stability of chaotic motions in quasi-integrable systems, Physica D 163, 1 (2002).

[74] A. Celletti, C. Falcolini, and U. Locatelli, On the breakdown threshold of invariant tori in four dimensional maps, Regular \& Chaotic Dynamics 9, 227 (2004).

[75] A. Bäcker and J. D. Meiss, Elliptic bubbles in Moser's $4 D$ quadratic map: The quadfurcation, SIAM J. Appl. Dyn. Syst. 19, 442 (2020).

[76] S. Lange, A. Bäcker, and R. Ketzmerick, What is the mechanism of power-law distributed Poincaré recurrences in higher-dimensional systems?, EPL 116, 30002 (2016).

[77] S. Anastassiou, T. Bountis, and A. Bäcker, Homoclinic points of $2 D$ and $4 D$ maps via the parametrization method, Nonlinearity 30, 3799 (2017).

[78] M. Firmbach, S. Lange, R. Ketzmerick, and A. Bäcker, Three-dimensional billiards: Visualization of regular structures and trapping of chaotic trajectories, Phys. Rev. E 98, 022214 (2018).

[79] C. C. Martens, M. J. Davis, and G. S. Ezra, Local frequency analysis of chaotic motion in multidimensional systems: Energy transport and bottlenecks in planar OCS, Chem. Phys. Lett. 142, 519 (1987).

[80] R. Bartolini, A. Bazzani, M. Giovannozzi, W. Scandale, and E. Todesco, Tune evaluation in simulations and experiments, Part. Accel. 52, 147 (1996).

[81] Ch. Skokos, G. A. Gottwald, and J. Laskar (editors) Chaos Detection and Predictability, volume 915 of Lecture Notes in Physics, Springer Berlin Heidelberg (2016).

[82] M. Born, The Mechanics of the Atom, G. Bell \& Sons, Ltd., London (1927).

[83] P. Gaspard and S. A. Rice, Hamiltonian mapping models of molecular fragmentation, J. Phys. Chem. 93, 6947 (1989).

[84] T. Schilling, 4D phase space and escape in van der Waals molecules, Masterthesis, Technische Universität Dresden, Fachrichtung Physik (2017).

[85] À. Haro, M. Canadell, J.-L. Figueras, A. Luque, and J.M. Mondelo, The Parameterization method for invari- ant manifolds: from rigorous results to effective computations, volume 195 of Applied Mathematical Sciences, Springer International Publishing (2016).

[86] J. Gonzalez and J. D. Mireles James, High-order parameterization of stable/unstable manifolds for long periodic orbits of maps, SIAM J. Appl. Dyn. Syst. 16, 1748 (2017).

[87] R. S. MacKay, J. D. Meiss, and I. C. Percival, Transport in Hamiltonian systems, Physica D 13, 55 (1984).

[88] V. Rom-Kedar and S. Wiggins, Transport in twodimensional maps, Arch. Rational Mech. Anal. 109, 239 (1990).

[89] J. D. Meiss, Symplectic maps, variational principles, and transport, Rev. Mod. Phys. 64, 795 (1992).

[90] J. D. Meiss, Thirty years of turnstiles and transport, Chaos 25, 097602 (2015).

[91] W. Bauer and G. F. Bertsch, Decay of ordered and chaotic systems, Phys. Rev. Lett. 65, 2213 (1990).

[92] M. Ding, T. Bountis, and E. Ott, Algebraic escape in higher dimensional Hamiltonian systems, Phys. Lett. A 151, 395 (1990).

[93] B. V. Chirikov and V. V. Vecheslavov, Theory of fast Arnold diffusion in many-frequency systems, J. Stat. Phys. 71, 243 (1993).

[94] D. L. Shepelyansky, Poincaré recurrences in Hamiltonian systems with a few degrees of freedom, Phys. Rev. E 82, 055202(R) (2010).

[95] P. Ramachandran and G. Varoquaux, Mayavi: 3D visualization of scientific data, Comput. Sci. Eng. 13, 40 (2011).

[96] Y. You, E. J. Kostelich, and J. A. Yorke, Calculating stable and unstable manifolds, Int. J. Bifurcation Chaos 1, 605 (1991).

[97] D. Hobson, An efficient method for computing invariant manifolds of planar maps, J. Comput. Phys. 104, 14 (1993).

[98] E. J. Kostelich, J. Yorke, and Z. You, Plotting stable manifolds: error estimates and noninvertible maps, Physica D 93, 210 (1996).

[99] R. H. Goodman and J. K. Wróbel, High-order bisection method for computing invariant manifolds of twodimensional maps, Int. J. Bifurcation Chaos 21, 2017 (2011).

[100] J. K. Wróbel and R. H. Goodman, High-order adaptive method for computing two-dimensional invariant manifolds of three-dimensional maps, Commun. Nonlinear Sci. Numer. Simulat. 18, 1734 (2013).

[101] C. Efthymiopoulos, G. Contopoulos, and M. Katsanikas, Analytical invariant manifolds near unstable points and the structure of chaos, Celest. Mech. Dyn. Astron. 119, 331 (2014).

[102] R. D. Neidinger, An efficient method for the numerical evaluation of partial derivatives of arbitrary order, ACM Trans. Math. Softw. 18, 159 (1992).

[103] V. O. E. Kjelseth, Efficient Calculation of Derivatives using Automatic Differentiation, Masterthesis, University of Oslo (2014). 